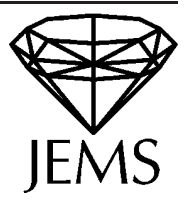

\title{
Asymptotic behaviour and the moduli space of doubly-periodic instantons
}

Received June 8, 2000 / final version received February 1, 2001

Published online April 3, 2001 - (c) Springer-Verlag \& EMS 2001

Abstract. We study doubly-periodic instantons, i.e. instantons on the product of a 1-dimensional complex torus $T$ with a complex line $\mathbb{C}$, with quadratic curvature decay. We determine the asymptotic behaviour of these instantons, constructing new asymptotic invariants. We show that the underlying holomorphic bundle extends to $T \times \mathbb{P}^{1}$. The converse statement is also true, namely a holomorphic bundle on $T \times \mathbb{P}^{1}$ which is flat on the torus at infinity, and satisfies a stability condition, comes from a doubly-periodic instanton. Finally, we study the hyperkähler geometry of the moduli space of doubly-periodic instantons, and prove that the Nahm transform previously defined by the second author is a hyperkähler isometry with the moduli space of certain meromorphic Higgs bundles on the dual torus.

\section{Contents}

I Connections with quadratic curvature decay . . . . . . . . . . . . . . . . . 340

1 Limit flat connection . . . . . . . . . . . . . . . . . . . 341

2 The linear problem .................. 344

3 Existence of a Coulomb gauge . . . . . . . . . . . . . . . . . . . . 349

II Instantons, holomorphic bundles, and the moduli space . . . . . . . . . . . . . 352

4 Asymptotic behaviour: proof of theorem $0.1 \ldots \ldots 352$

5 Holomorphic extension . . . . . . . . . . . . . . . . . . 353

6 Moduli spaces . . . . . . . . . . . . . . . . . . . . . . . . . . 360

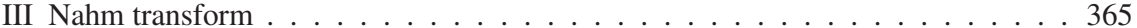

7 Asymptotic parameters . . . . . . . . . . . . . . . . . . 366

8 The hyperkähler property . . . . . . . . . . . . . . . . . . . 370

\section{Introduction and statement of the results}

The aim of this paper is to understand the analytical properties of certain finite energy solutions of the Yang-Mills anti-self-dual equations over $T \times \mathbb{C}$. These so-called extensible doubly-periodic instantons have been studied by the second

O. Biquard: Institut de Recherche Mathématique Avancée, Université Louis Pasteur et CNRS, 7 rue René Descartes, 67084 Strasbourg Cedex, France

M. Jardim: University of Pennsylvania, Department of Mathematics, 209 South 33rd St., Philadelphia, PA 19104-6395, USA

Mathematics Subject Classification (2000): 53C07, 53C26 
author in [12-14], where they were shown to be equivalent to certain singular solutions of Hitchin's equations over an elliptic curve via a construction known as the Nahm transform. The present paper grew from questions raised in the works mentioned above.

More precisely, consider an $S U_{2}$ bundle $E \rightarrow T \times \mathbb{C}$. The instanton connections $A$ considered in $[12,13]$ satisfied the following hypothesis:

1. quadratic curvature decay: $\left|F_{A}\right|=O\left(r^{-2}\right)$ with respect to the Euclidean metric on $T \times \mathbb{C}$;

2. extensibility: there is a holomorphic rank two vector bundle $\mathcal{E} \rightarrow T \times \mathbb{P}^{1}$ with trivial determinant such that $\left.\mathcal{E}\right|_{T \times\left(\mathbb{P}^{1} \backslash\{\infty\}\right)} \simeq\left(E, \bar{\partial}_{A}\right)$, where $\bar{\partial}_{A}$ is the holomorphic structure on $E$ induced by $A$;

where $w$ is a coordinate in the complex line, and by the notation $O\left(|w|^{\gamma}\right)$ we mean the set of functions on $\mathbb{C}$ such that: $\lim _{|w| \rightarrow \infty}|f(w)| /|w|^{\gamma}<\infty$.

One of the goals of this paper is to prove that the technical hypothesis of extensibility is actually a consequence of the anti-self-duality equation, and more generally to understand completely the behaviour at infinity of all instantons with quadratic curvature decay.

Recently, physicists have also been increasingly interested in doubly-periodic instantons. They were studied by Kapustin \& Sethi in the context of certain string theories [11]. In a different direction, González-Arroyo \& Montero among others have been studying various periodic instantons in the context of Quantum Field Theory in the lattice (see in particular [9]). Finally, an attempt to construct explicit analytic solutions has been made by Ford, Pawlowski, Tok and Wipf in [6].

Model solutions. Special solutions of the anti-self-duality equations may be obtained by restricting to torus invariant connections. Such instantons come from solutions $(B, \psi)$ of Hitchin's equations on $\mathbb{C}$

$$
\left\{\begin{array}{l}
F_{B}+\left[\psi, \psi^{*}\right]=0 \\
\bar{\partial}_{B} \psi=0
\end{array}\right.
$$

in the following way. Recall that $B$ is a $S U_{2}$-connection on $\mathbb{C}$, and $\psi$ is a $(1,0)$-form with values in $\mathfrak{s l}_{2}$. Let $\psi=\frac{1}{2}\left(\psi_{0}+i \psi_{1}\right) d w$, and consider the connection (where $x$ and $y$ are coordinates on $T$ ):

$$
A_{0}=B+\psi_{0} d x+\psi_{1} d y
$$

which is a torus invariant instanton. Assuming that $\left|F_{A_{0}}\right|=O\left(r^{-2}\right)$, the asymptotic behaviour of solutions $(B, \psi)$ is given by one of the following models:

$$
\begin{array}{ccc}
B=d & \psi=\left(\begin{array}{cc}
\lambda & 0 \\
0 & -\lambda
\end{array}\right) d w \\
B=d+\left(\begin{array}{cc}
\alpha & 0 \\
0 & -\alpha
\end{array}\right) i d \theta & \psi=\left(\begin{array}{cc}
\mu & 0 \\
0 & -\mu
\end{array}\right) \frac{d w}{w} \\
B=d+\left(\begin{array}{cc}
-1 & 0 \\
0 & 1
\end{array}\right) \frac{i d \theta}{\ln r^{2}} & \psi=\left(\begin{array}{cc}
0 & 1 \\
0 & 0
\end{array}\right) \frac{d w}{w \ln r^{2}}
\end{array}
$$


where $\lambda, \mu \in \mathbb{C}$ and $-\frac{1}{2} \leq \alpha<\frac{1}{2}$. The solutions of examples (1) \& (2) can be superimposed, and such superpositions are called the semisimple solutions. On the other hand, solutions of example (3) cannot be superimposed with the others; these are called the nilpotent solutions, and can only exist when $\lambda=\mu=\alpha=0$. The torus invariant instanton is then given by, in the semisimple case:

$$
A_{0}=d+i\left(\begin{array}{cc}
a_{0} & 0 \\
0 & -a_{0}
\end{array}\right)
$$

with

$$
a_{0}=\lambda_{1} d x+\lambda_{2} d y+\left(\mu_{1} \cos \theta-\mu_{2} \sin \theta\right) \frac{d x}{r}+\left(\mu_{1} \sin \theta+\mu_{2} \cos \theta\right) \frac{d y}{r}+\alpha d \theta ;
$$

while in the nilpotent case, we have:

$$
A_{0}=d+i\left(\begin{array}{cc}
-1 & 0 \\
0 & 1
\end{array}\right) \frac{d \theta}{\ln r^{2}}+\frac{1}{r \ln r^{2}}\left(\begin{array}{cc}
0 & e^{-i \theta}(d x-i d y) \\
-e^{i \theta}(d x+i d y) & 0
\end{array}\right)
$$

and note that the curvature is $O\left(r^{-2}\left|\ln r^{2}\right|^{-2}\right)$.

Remark that the connection $A_{0}$ has a flat limit over the torus at infinity,

$$
d+i\left(\begin{array}{cc}
\lambda_{1} d x+\lambda_{2} d y & 0 \\
0 & -\lambda_{1} d x-\lambda_{2} d y
\end{array}\right)
$$

and one can prove that such flat limit for a connection $A$ exists as soon as $\left|F_{A}\right|=$ $O\left(r^{-1-\epsilon}\right)$; the flat limit underlies a holomorphic vector bundle $L_{\xi_{0}} \oplus L_{-\xi_{0}}$, where the elements of the dual torus $\pm \xi_{0} \in \hat{T}$ are called the asymptotic states of the connection.

We show that the three standard examples above completely describe the behaviour at infinity of doubly-periodic instantons with quadratic curvature decay:

Theorem 0.1. Let $A$ be a doubly-periodic instanton with curvature $O\left(r^{-2}\right)$. Then there is a gauge near infinity such that

$$
A=A_{0}+a,
$$

where $A_{0}$ is one of the previous models, and, for some $\delta>0$, in the semisimple case:

$$
|a|=O\left(\frac{1}{r^{1+\delta}}\right), \quad\left|\nabla_{A_{0}} a\right|=O\left(\frac{1}{r^{2+\delta}}\right) ;
$$

in the nilpotent case:

$$
|a|=O\left(\frac{1}{r(\ln r)^{1+\delta}}\right), \quad\left|\nabla_{A_{0}} a\right|=O\left(\frac{1}{r^{2}(\ln r)^{2+\delta}}\right) .
$$


In the case where the limit at infinity of $A$ is non trivial, one can prove the theorem under the weaker assumption that the curvature is $O\left(r^{-1-\epsilon}\right)$; this condition is very close to the finite energy condition, and it is natural to suppose that the theorem actually describes the behaviour of all finite energy instantons. The instantons we will use (for example, those coming from the inverse Nahm transform) have quadratic curvature decay, so that this hypothesis is sufficient for our applications.

The theorem, to be proved in Sect. 4, provides a complete characterization of the instanton parameters which are invariant under $L^{2}$ deformations. The parameter $\lambda$ is equivalent to the asymptotic states $\pm \xi_{0}$. The two remaining parameters are new: $\alpha$ is called the limiting holonomy of the instanton $A$, while $\mu$ is called the residue. The motivation for the latter nomenclature will be made clear latter on. Notice that, in contrast with the instanton number (see below) and the asymptotic states, the limiting holonomy and the residues are defined only for anti-self-dual connections.

Instantons and holomorphic bundles. We are now ready to state our second main result, which in particular solves the extensibility problem. Recall [13] that the instanton number $k$ of the doubly-periodic instanton $A$ is defined by the formula:

$$
k=\frac{1}{8 \pi^{2}} \int_{T \times \mathbb{C}}\left|F_{A}\right|^{2}
$$

as usual.

Theorem 0.2. There is a 1-1 correspondence between the following objects:

- $S U_{2}$-doubly-periodic instanton connections with quadratic curvature decay and fixed asymptotic parameters $\left(k, \pm \xi_{0}, \alpha\right)$;

- $\alpha$-stable, rank two holomorphic vector bundles $\mathcal{E} \rightarrow T \times \mathbb{P}^{1}$ with trivial determinant such that $c_{2}(\mathcal{E})=k$ and $\left.\mathcal{E}\right|_{T \times\{\infty\}}=L_{\xi_{0}} \oplus L_{-\xi_{0}}$.

The stability condition of the statement is a variant of the stability condition for parabolic bundles; the degree is calculated with respect to a non ample class (the fundamental class of the torus). The precise definition will be given in Sect. 5, where this result is proved.

In a broader context, theorem 0.2 can be seen as the analog of Donaldson's correspondence between instantons on $\mathbb{R}^{4}$ and framed holomorphic bundles over $\mathbb{P}^{2}[7,5]$. In this last case, no stability condition is needed in order to produce an instanton, while in the case of a compact surface, stability (with respect to an ample class) is necessary. Thus, in some sense, our stability criterion goes midway between these two situations.

Moduli space. We then pass to the analytical construction of the moduli space of doubly-periodic instantons. We prove:

Theorem 0.3. The moduli space of doubly-periodic instantons with fixed instanton number $k$ and asymptotic parameters $\left( \pm \xi_{0}, \alpha, \mu\right)$ is a smooth hyperkähler manifold of real dimension $8 k-4$. 
Of course, this theorem is interesting only if the moduli space is not empty. Fortunately, as mentioned in [12], existence of doubly-periodic instantons for generic values of the parameters $\left(k, \pm \xi_{0}, \alpha, \mu\right)$ is guaranteed via the Nahm transform (see below) of meromorphic Higgs bundles over $\hat{T}$, whose existence follows from Simpson [22] among others; theorem 0.1 puts these instantons in our moduli spaces. Another equivalent, probably more direct, way for guaranteeing existence is of course theorem 0.2. See also Sect. 5 for some cases where the moduli space is empty, and Sect. 6 for a description of the $k=1$ moduli space.

Nahm transform. Finally we revisit the Nahm transform of doubly-periodic instantons defined in [13] with two main objectives in mind. Before explaining what these objectives are, let us say a few words about the Nahm transform.

Here we restrict to the semisimple case, since Nahm transform was defined only in this case. Recall from [13] (see also part III) the Nahm Transform is a 1-1 correspondence between irreducible, doubly-periodic instantons and certain meromorphic Higgs pairs $(V, \Phi)$ with harmonic metric over the dual torus $\hat{T}$. The rank of $V$ is given by the instanton number, and its degree is -2 . The Higgs field $\Phi$ has simple poles at the two points corresponding to the asymptotic states $\pm \xi_{0}$. Moreover, $\Phi$ has semisimple residues of rank one if $\xi_{0} \neq-\xi_{0}$, and rank two otherwise. We denote by $\operatorname{Res} \Phi\left( \pm \xi_{0}\right)$ the residue of the Higgs field at the singular point $\pm \xi_{0}$.

Thus, it is natural to ask how are the new asymptotic parameters defined by theorem 0.1 interpreted in terms of the Nahm transformed Higgs pair. This question in answered in Sect. 7. As expected from the general principle Nahm transform is a non-linear Fourier transform, the asymptotic behaviour is converted into singularity behaviour. Before stating the result, let us fix some notations: at the puncture $\pm \xi_{0}$ we have a decomposition

$$
V=B \oplus R
$$

where $B=\operatorname{ker}(\operatorname{Res} \Phi)$ and $R$ is the 1 -dimensional eigenspace of $\operatorname{Res} \Phi$; the eigenspaces of $\Phi$ enable to extend this decomposition near the puncture as a local holomorphic splitting of $V$.

Theorem 0.4. Let A be a doubly-periodic instanton with limiting holonomy $\alpha$ and residue $\mu$; let $(V, \Phi)$ be its Nahm transformed Higgs pair.

Then the unique nonzero eigenvalue of $\operatorname{Res}_{ \pm \xi_{0}} \Phi$ is given by $\pm \mu$. Moreover, the harmonic metric on $V$ remains bounded on $B$, but behaves like $\left|\xi \pm \xi_{0}\right|^{1 \pm \alpha}$ on $R$.

Note that the sum of the degree of $V$, that is -2 , and of the weights $1 \pm \alpha$, equals 0 , as must be for a solution of Hitchin's selfduality equations (see also remark 7.5 for more informations on the induced stable parabolic Higgs bundle).

The monodromy of the connection $B$ near the punctures is semisimple, with only one nontrivial eigenvalue $\exp (\mp 2 \pi i \alpha)$ on $R$ (or two if $\xi_{0}=-\xi_{0}$ ).

It is well known that the moduli space of Higgs pairs on a Riemann surface is hyperkähler [10]; for the moduli space of Higgs pairs with fixed singularities at the punctures, this follows from [3]. The second goal can now be summarized in our last result: 
Theorem 0.5. The Nahm transform of doubly-periodic instantons is a hyperkähler isometry.

Note that similar results have been proved for the other well-known examples of Nahm transform: the ADHM construction, see [17]; the duality between monopoles and solutions of Nahm equations, see [18]; and the Fourier-Mukai transform of instantons over 4-tori, see [4]. Indeed, it is reasonable to expect that such result holds for any Nahm transform.

Outline. The paper is divided in three parts. The first part is technical: we study the asymptotic behaviour of connections on $E$ with quadratic curvature decay, but which are not necessarily anti-self-dual; the technical goal is the construction of a partial Coulomb gauge (theorem 0.6). In the second part, we obtain theorems $0.1,0.2$ and 0.3 . Finally, the third part deals with the Nahm transform aspects of the paper.

\section{Connections with quadratic curvature decay}

In this part, we study the behaviour at infinity of (not necessarily anti-self-dual) connections with quadratic curvature decay on a $S U_{2}$-bundle $E$ on $T \times \mathbb{R}^{2}$. Such connections will have a limit flat connection $\Gamma$ on the torus at infinity $T_{\infty}$, which decomposes $\left.E\right|_{T_{\infty}}$ as a sum of two flat line bundles $L_{\xi_{0}} \oplus L_{-\xi_{0}}$; when $L_{\xi_{0}}^{2}=0$, we can reduce to the case where $L_{\xi_{0}}=0$ by globally tensoring $E$ with $L_{\xi_{0}}$; therefore we will always suppose that

$$
\text { either } L_{\xi_{0}}^{2} \neq 0, \quad \text { or } L_{\xi_{0}}=0 \text {. }
$$

Over any torus $T$, we consider the orthogonal decomposition of the space of $L^{2}$-sections of $E$ :

$$
L^{2}(T, \operatorname{End}(E))=\left(\operatorname{ker} \nabla_{\Gamma}\right) \oplus\left(\operatorname{ker} \nabla_{\Gamma}\right)^{\perp},
$$

and we decompose accordingly any section $u$ of $\operatorname{End}(E)$ as

$$
u=u_{\Gamma}+u_{\perp}
$$

If we write explicitly $\Gamma=d+\gamma$, with

$$
\gamma=\left(\begin{array}{cc}
\lambda_{1} & 0 \\
0 & -\lambda_{1}
\end{array}\right) i d x+\left(\begin{array}{cc}
\lambda_{2} & 0 \\
0 & -\lambda_{2}
\end{array}\right) i d y
$$

then, in view of (4), $\operatorname{ker} \nabla_{\Gamma}$ is described as the $T$-invariant sections of $\operatorname{ker} \gamma$; if $\gamma$ is nontrivial, these are reduced to $T$-invariant diagonal matrices.

The technical goal of this part is a partial Coulomb gauge on the $a_{\perp}$ part of a connection $A=\Gamma+a$ with curvature $O\left(r^{-2}\right)$. More precisely, let $V_{R} \subset \mathbb{C}$ denote the complement of a disc of radius $R$ centered at the origin. 
Theorem 0.6. Given a constant $\eta>0$, there exists $R$ sufficiently large such that if $A$ is a doubly-periodic connection satisfying $\sup _{r \geq R}\left(r^{2}\left|F_{A}\right|\right) \leq \eta$, then there is a gauge $g$ on $T \times V_{R}$ such that $g(A)=\Gamma+a_{\Gamma}+a_{\perp}$, with:

$$
\begin{aligned}
& \text { (i) } d_{\Gamma+a_{\Gamma}}^{*} a_{\perp}=0 ; \\
& \text { (ii) } \left.\partial_{r}\right\lrcorner a_{\perp}(r=R)=0 ; \\
& \text { (iii) }\left\|r^{2} F_{\Gamma+a_{\Gamma}}\right\|_{C^{0}}+\left\|r^{2-\epsilon} a_{\perp}\right\|_{C^{0}} \leq C \cdot\left\|r^{2} F_{A}\right\|_{C^{0}} .
\end{aligned}
$$

Note that gauge transformations $g \in \operatorname{ker} \nabla_{\Gamma}$ preserve the Coulomb gauge constructed in this theorem. This kind of partial gauge fixing reminds of Råde's fibered Hodge gauge [20].

Remark 0.7. Actually, if $\Gamma$ is nontrivial, the proof gives a Coulomb gauge under a weaker bound on the curvature, namely $|F|=O\left(r^{-(1+\epsilon)}\right)$; this condition is very close to the finite energy condition, since $r^{-\delta}$ is in $L^{2}$ when $\delta>1$.

\section{Limit flat connection}

Our first task is to establish the existence of a flat limit connection $\Gamma$ for every connection $A$ with quadratic curvature decay:

Proposition 1.1. Suppose that the connection $A$ on $T \times \mathbb{R}^{2}$ satisfies

$$
\left|F_{A}\right| \leq \frac{c_{1}}{r^{2}} .
$$

Then A has a flat limit $\Gamma$ on $T$ at infinity, and there exists a sequence of connections $A_{j}$, such that

1. $\left|F_{A_{j}}\right| \leq c_{2} / r^{2}$;

2. $A_{j}$ is gauge equivalent to $A$ on $\{r \leq j\}$;

3. $A_{j}=\Gamma$ on $\{r \geq 2 j\}$.

Remark 1.2. This proposition remains true if the curvature is $O\left(r^{-(1+\epsilon)}\right)$.

Proof. We begin by proving the existence of the flat limit $\Gamma$. Take a radial gauge

$$
A=d+a_{\theta} d \theta+a_{x} d x+a_{y} d y
$$

for $A$; from the bound on the curvature, we deduce

$$
\left|\partial_{r} a_{x}\right|+\left|\partial_{r} a_{y}\right|=O\left(r^{-2}\right), \quad\left|\partial_{r} a_{\theta}\right|=O\left(r^{-1}\right) ;
$$

from this we deduce that $a_{x}$ and $a_{y}$ have limits $a_{x}^{\infty}(\theta, x, y)$ and $a_{y}^{\infty}(\theta, x, y)$ when $r$ goes to infinity; moreover, the bound on the curvature implies that for each $\theta$, the connection $d+a_{x}^{\infty}(\theta) d x+a_{y}^{\infty}(\theta) d y$ is flat on $T$. It remains to see that it is independent of $\theta$ : for this we pick a base point in $T=S^{1} \times S^{1}$ and prove that the monodromies along the two circles remain conjugate when $\theta$ varies; this is a consequence of the bound on the curvature and the following lemma (see for example [1, lemma 1]): 
Lemma 1.3. Suppose we have a connection $A$ on $[0,1] \times S^{1}$, and $m(t)$ is the monodromy of A along the circle $\{t\} \times S^{1}$; note $h(t)$ the parallel transport from the point $(0,0)$ to the point $(t, 0)$; then

$$
\left|\partial_{t}\left(h(t)^{-1} m(t) h(t)\right)\right| \leq \int_{\{t\} \times S^{1}}\left|F_{A}\right| .
$$

Therefore we have constructed a flat limit $\Gamma$ on $T$ for the connection $A$. Now pass to the approximation statement.

Claim. On $\{r\} \times S^{1} \times T$, there exists a gauge so that $A=\Gamma+a,|a| \leq c / r$.

This statement (a $C^{0}$ gauge only), can be proven by elementary means and is left to the reader. Now, we extend radially this gauge on $\{j\} \times S^{1} \times T$ to $[j, 2 j] \times S^{1} \times T$, and the bounds (8) imply that $A=\Gamma+a$ with still $|a| \leq c / r$ on $[j, 2 j]$; then we choose a cutoff function $\chi=\chi(r)$ so that

$$
\chi(r \leq j)=1, \quad \chi(r \geq 2 j)=0, \quad\left|\partial_{r} \chi\right| \leq 2 / j,
$$

and define a connection $A_{j}$ by

$$
\begin{array}{ll}
r \leq j, & A_{j}=A, \\
r \geq j, & A_{j}=\Gamma+\chi a ;
\end{array}
$$

on $r \geq j$, the curvature of $A_{j}$ is

$$
F_{A_{j}}=\chi F_{A}+d \chi \wedge a+\left(\chi^{2}-\chi\right) a \wedge a
$$

and this remains bounded by $c / r^{2}$ on $[j, 2 j]$, which means that $\left|F_{A_{j}}\right|$ is uniformly bounded by $c / r^{2}$.

Remark 1.4. Actually, it is not difficult to go a bit further and to prove that there is a global gauge in which $A=\Gamma+a$ and $|a|=O(\ln r / r)$; this gives a result used without proof in [13]. Of course, the result will also be a consequence of theorem 0.6.

In the case of a torus invariant connection, we need a stronger statement.

Proposition 1.5. Under the hypotheses of proposition 1.1, if $A=d+a$ with $a=a_{\Gamma}$ (in particular $A$ is torus invariant), then there is a gauge such that

$$
A=\Gamma+a+b,
$$

where $d+a$ is a connection on $\mathbb{R}^{2}$ and $b=b_{x} d x+b_{y} d y$ a 1-form along $T$, satisfying

and

$$
|b| \leq \frac{c_{3}}{r}, \quad\left|\nabla_{\Gamma+a} b\right| \leq \frac{c_{2}}{r^{2}},
$$

$$
a=i\left(\begin{array}{cc}
\alpha(r) & 0 \\
0 & -\alpha(r)
\end{array}\right) d \theta+a^{\prime}
$$

with

$$
\left|\partial_{r} \alpha\right|+\left|a^{\prime}\right| \leq c_{3} / r, \quad \sup _{j} j^{2-2 / p}\left\|\nabla a^{\prime}\right\|_{L^{p}(j \leq r \leq 2 j)} \leq c_{3} .
$$


The meaning is that we want a gauge with not only a $C^{0}$ bound, but also a $C^{1}$ bound; actually this is not possible (because elliptic regularity does not hold in $C^{k}$ spaces) and this explains why we use $L^{p}$ derivatives instead. So the proposition must be considered as a regularization of the connection. The standard way to obtain this is to use Hodge gauges in order for the curvature to become an elliptic equation: locally Uhlenbeck's theorem provides the required statement, but the gluing is not easy, especially on a non simply connected manifold. We present here a proof based on the following lemma, which is a consequence of the Hodge gauge constructed in [1, theorem 1]:

Lemma 1.6. Any connection A on $[0,1] \times S^{1}$, with $\left\|F_{A}\right\|_{L^{p}}$ sufficiently small, is gauge equivalent to a connection $d+i \alpha d \theta+a$ with $\|a\|_{L^{1, p}} \leq c\left\|F_{A}\right\|_{L^{p}}$, where $\alpha$ is a diagonal matrix, with coefficients in $[0,1[$, such that $\exp (-2 \pi i \alpha)$ is the monodromy of $A$ along the circle $\{0\} \times S^{1}$.

Proof of proposition 1.5. If $A$ is torus invariant, then a torus invariant gauge transformation $g$ acts on $b$ only by $g g^{-1}$, and the bounds on the curvature immediately imply the required bounds on $b$. Therefore, we are reduced to look at a connection $d+a$ on $\mathbb{R}^{2}$.

Now note that the region $r \geq 1$ is conformally equivalent to the half-cylinder $\mathbb{R}_{+} \times S^{1}$ (with coordinate $t=\ln r$ ); in the rest of the proof we will use only the flat metric on the cylinder. The bound on the curvature becomes $\left|F_{A}\right| \leq c_{2}$; eventually pulling back $A$ using the transformation $t \rightarrow \lambda t$ with $\lambda$ sufficiently small, we may suppose that $c_{2}$ is very small. This means that we are now able to use lemma 1.6, for some $p$ very big, to produce on each $[j-1, j+1] \times S^{1}$ a gauge $g_{j}$ so that

$$
g_{j}(A)=d+i \alpha_{j} d \theta+a_{j}, \quad\left\|a_{j}\right\|_{L^{p}}+\left\|\left(\nabla+i \alpha_{j} d \theta\right) a_{j}\right\|_{L^{p}} \leq c c_{2} .
$$

We perform recursively diagonal gauge transformations with coefficients of type $\exp (i k \theta)(k$ integer) so that we have

$$
\left|\alpha_{j+1}-\alpha_{j}\right|<c_{2}
$$

this is possible because of lemma 1.3, and the operation does not affect the bound on $a_{j}$ (but we have only $\left|\alpha_{j}\right| \leq c_{2} j$ ). We want to glue together these local gauges: the transition $h_{j}=g_{j+1} \cdot g_{j}^{-1}$ satisfies

$$
d h_{j}+\left[i \alpha_{j} d \theta, h_{j}\right]=h_{j} \cdot a_{j}-\left(\left(\alpha_{j+1}-\alpha_{j}\right) i d \theta+a_{j+1}\right) \cdot h_{j} ;
$$

the RHS is controled by $c c_{2}$, and this implies that $h_{j}$ is very close to some $\tilde{h}_{j}(\theta)$ in the kernel of $d+i \alpha_{j} d \theta$; replacing $g_{j+1}$ by $\tilde{h}_{j} \cdot g_{j+1}$, we now may suppose that the transition $g_{j+1} \cdot g_{j}^{-1}$ is close to the identity (in $L^{2, p}$ norm), and a standard argument now enables us to glue together all these gauges: for a similar argument, see [1, pages 447-8]. If we choose diagonal matrices $\alpha(t)$ so that

$$
\alpha(j)=\alpha_{j}, \quad\left|\partial_{t} \alpha\right| \leq 2 c_{2},
$$

we finally get a gauge $d+i \alpha(t) d \theta+a^{\prime}$, with

$$
\left\|a^{\prime}\right\|_{L^{p}([j-1, j+1])}+\left\|(\nabla+i \alpha(t) d \theta) a^{\prime}\right\|_{L^{p}([j-1, j+1])} \leq c c_{2} .
$$


Sobolev embedding implies that $\left\|a^{\prime}\right\|_{C^{0}}$ is controled as well; translating back these bounds in the metric of $\mathbb{R}^{2}$, we get the proposition.

Remark 1.7. The proof of proposition 1.5 becomes certainly easier if $A$ is abelian (which is the case if the limit $\Gamma$ is regular), since in this case, it is easy to produce a global Hodge gauge.

Remark 1.8. In general, we are unable to prove proposition 1.5 if the curvature is only $O\left(r^{-(1+\epsilon)}\right)$ : this is because, in order to get a controled gauge on $\mathbb{R}_{+} \times S^{1}$, we need the curvature to be bounded; if $\Gamma$ is nontrivial, the problem becomes abelian, and then it is easy to construct a global Hodge gauge on $\mathbb{R}_{+} \times S^{1}$, from which the proposition follows easily (and one gets a bound in $O\left(r^{-\epsilon}\right)$ on $a$ ).

\section{The linear problem}

In this section we study the linear analysis on the $\left(\operatorname{ker} \nabla_{\Gamma}\right)_{\perp}$ part for the Laplacian operator $d_{\Gamma}^{*} d_{\Gamma}$ acting on 0 -forms and the deformation operator $d_{\Gamma}^{+}+d_{\Gamma}^{*}$ acting on 1-forms, with fixed boundary conditions.

For this analysis, we will use the Sobolev spaces $L^{p, k}$ of functions with $k$ derivatives in $L^{p}$; the weighted Sobolev spaces $L_{\delta}^{p, k}$ of functions $f$ such that $\left(1+r^{2}\right)^{\delta / 2} f \in L^{p, k}$.

The basis of the analysis is the following simple lemma, which is an immediate consequence of the decomposition (5).

Lemma 2.1. There is a constant $c$, depending on $p$, such that on each torus $T$, for any section $u$ of End $(E)$, we have:

$$
\int_{T}\left|\nabla_{\Gamma} u_{\perp}\right|^{p} \geq c \int_{T}\left|u_{\perp}\right|^{p}
$$

Analysis on 0-forms

Lemma 2.2. The Neumann problem on sections of End $(E)_{\perp}$ on $r \geq R$,

$$
\left\{\begin{array}{l}
\Delta_{\Gamma} u=v \\
\partial_{r} u(r=R)=0
\end{array}\right.
$$

is an isomorphism $L_{\delta}^{2,2} \rightarrow L_{\delta}^{2}$.

Proof. The solution $u$ of the Neumann problem is obtained by minimizing the functional

$$
\int \frac{1}{2}\left|\nabla_{\Gamma} u\right|^{2}-\langle u, v\rangle
$$

in the space $L^{1,2}$; the minimization is possible because of the estimate (9); local elliptic regularity gives that the $L^{1,2}$-solution actually lives in $L^{2,2}$, and this gives the statement when there is no weight. 
In the case we have a weight $\delta$, the following estimate holds:

$$
\begin{aligned}
\int\left\langle\Delta_{\Gamma} u, u\right\rangle r^{2 \delta} & =\int\left|\nabla_{\Gamma} u\right|^{2} r^{2 \delta}+2 \frac{\delta}{r}\left\langle\nabla_{\partial_{r}} u, u\right\rangle r^{2 \delta} \\
& \geq \int\left(1-\frac{\delta}{r}\right)\left|\nabla_{\Gamma} u\right|^{2} r^{2 \delta}-\frac{\delta}{r}|u|^{2} r^{2 \delta}
\end{aligned}
$$

and using (9) we get, if $R$ is large enough,

$$
\begin{aligned}
\left\|\Delta_{\Gamma} u\right\|_{L_{\delta}^{2}}\|u\|_{L_{\delta}^{2}} & \geq \int\left\langle\Delta_{\Gamma} u, u\right\rangle r^{2 \delta} \\
& \geq C\|u\|_{L_{\delta}^{2}}^{2}
\end{aligned}
$$

and therefore

$$
C\|u\|_{L_{\delta}^{2}} \leq\left\|\Delta_{\Gamma} u\right\|_{L_{\delta}^{2}}
$$

which proves that the isomorphism persists between weighted $L^{2}$-spaces, at least if $R$ is large enough.

This would be enough for our applications, but one can prove easily that the statement remains true for any $R$ : because $\Delta_{\Gamma}$ is an isomorphism for $R$ big enough, it remains a Fredholm operator for any $R$ (just glue the inverse near infinity with a parametrix on the compact part); the index is locally constant and therefore does not depend on the weight $\delta$; this means that it is equal to the $L^{2}$-index, that is 0 ; now, because the $L^{2}$-kernel is zero, the $L_{\delta}^{2}$-kernel is zero if $\delta>0$; for general $\delta$, the kernel is the $L_{\delta}^{2}$-kernel, while the cokernel is the $L_{-\delta}^{2}$-kernel: as at least one of them is trivial and the index is 0 , both are trivial.

We now want to deduce the same result in $L^{p}$ spaces. We need an estimate on the solution of problem (10) when $v$ is $L^{p}$. After a conformal change in the Euclidean metric $g_{E}$, we can pass to the cusp metric $\left(r=e^{t}\right)$ :

$$
g_{C}=d t^{2}+d \theta^{2}+e^{2 t}\left(d x^{2}+d y^{2}\right)=\frac{1}{r^{2}} g_{E} .
$$

The operator $\Delta_{\Gamma}$ now has singular coefficients, but is basically of the type studied in [3], where Hölder and $L^{p}$ estimates are deduced from the $L^{2}$-estimates. Here, the same techniques lead to the desired result:

Lemma 2.3. The Neumann problem (10) for 0-forms on $r \geq R$ is an isomorphism $L_{\delta}^{2, p} \rightarrow L_{\delta}^{p}$ for all weights $\delta$.

Proof. For the convenience of the reader, we give here a sketch of proof for the statement, inspired from [3, Sect. 6], but written with respect to the Euclidean metric. The proof below works for $p>2$ (the case we will use), but the statement remains true for general $p$.

The first step is to give an elliptic estimate

$$
\|u\|_{L_{\delta}^{2, p}[r, 2 r]} \leq c\left(\left\|\Delta_{\Gamma} u\right\|_{L_{\delta}^{p}\left(\left[\frac{1}{2} r, 3 r\right]\right)}+\|u\|_{L_{\delta-1+2 / p}^{2}\left(\left[\frac{1}{2} r, 3 r\right]\right)}\right) .
$$


The weight

$$
\delta_{2}=\delta-1+2 / p
$$

chosen for the $L^{2}$ space corresponds to functions with the same order of decreasing in $r^{-\delta-2 / p}$ as in the weighted $L^{p}$ space, but actually the proof below will give more. In order to prove this, we remark that, if $\lambda_{1}$ and $\lambda_{2}$ are the real numbers defined by (7), and

$$
\Lambda_{i}=\left(\begin{array}{cc}
\lambda_{i} & 0 \\
0 & -\lambda_{i}
\end{array}\right)
$$

then one has $(\operatorname{Ad}(g)$ denoting the conjugation by $g)$

$$
\nabla_{\Gamma}=\operatorname{Ad}\left(e^{-i\left(\Lambda_{1} x+\Lambda_{2} y\right)}\right) \circ \nabla \circ \operatorname{Ad}\left(e^{i\left(\Lambda_{1} x+\Lambda_{2} y\right)}\right)
$$

so that if we consider $x$ and $y$ as coordinates on $\mathbb{R}^{2}$, the equation $\Delta_{\Gamma} u=v$ becomes equivalent to

$$
\Delta u^{\prime}=\operatorname{Ad}\left(e^{i\left(\Lambda_{1} x+\Lambda_{2} y\right)}\right) v, \quad u^{\prime}=\operatorname{Ad}\left(e^{i\left(\Lambda_{1} x+\Lambda_{2} y\right)}\right) u .
$$

In the domain $[1,2] \times S^{1} \times[-1,1]^{2} \subset \mathbb{R}^{2} \times \mathbb{R}^{2}$, we have an elliptic estimate

$$
\left\|u^{\prime}\right\|_{L^{2, p}} \leq c\left(\left\|u^{\prime}\right\|_{L^{2}}+\left\|\Delta u^{\prime}\right\|_{L^{p}}\right)
$$

which implies on the homothetic domain $[R, 2 R] \times S^{1} \times[-R, R]^{2} \subset \mathbb{R}^{2} \times \mathbb{R}^{2}$

$$
R^{2-4 / p}\left\|\nabla^{2} u^{\prime}\right\|_{L^{p}} \leq c\left(R^{-2}\left\|u^{\prime}\right\|_{L^{2}}+R^{2-4 / p}\left\|\Delta u^{\prime}\right\|_{L^{p}}\right)
$$

and therefore on $[R, 2 R] \times S^{1} \times T$

$$
R^{2-2 / p}\left\|\nabla_{\Gamma}^{2} u\right\|_{L^{p}} \leq c\left(R^{-1}\|u\|_{L^{2}}+R^{2-2 / p}\left\|\Delta_{\Gamma} u\right\|_{L^{p}}\right)
$$

which we can rewrite, still on $[R, 2 R] \times S^{1} \times T$,

$$
\left\|\nabla_{\Gamma}^{2} u\right\|_{L_{\delta}^{p}} \leq c\left(\|u\|_{L_{\delta-3+2 / p}^{2}}+\left\|\Delta_{\Gamma} u\right\|_{L_{\delta}^{p}}\right)
$$

now the estimate (9) implies

$$
\left\|\nabla_{\Gamma}^{k} u\right\|_{L^{p}} \geq c\|u\|_{L^{p}} ;
$$

this, with local elliptic regularity, gives the estimate (11).

The second step now consists in going from the $L^{2}$-estimates with weights to the $L^{p}$-estimate. Basically, one can do the following: let $P$ be the inverse obtained by the $L^{2}$-resolution; decompose

$$
v=\sum v_{i}
$$

where $v_{i}$ has support in $\exp (i / 2)<r<\exp (3 i)$; by the $L^{2}$-resolution for the weight $\delta_{2}$ defined by (12), one has

$$
\left\|P v_{i}\right\|_{L_{\delta_{2}}^{2}} \leq c\left\|v_{i}\right\|_{L_{\delta_{2}}^{2}} \leq c\left\|v_{i}\right\|_{L_{\delta}^{p}}
$$


on the other hand, we decompose similarly $u_{i}=P v_{i}$ as

$$
u_{i}=\sum_{j} u_{i j}
$$

and we note that the $L^{2}$ resolution gives the estimate

$$
\begin{aligned}
\left\|u_{i j}\right\|_{L_{\delta_{2}}^{2}} & \leq c e^{-\epsilon j}\left\|u_{i j}\right\|_{L_{\delta_{2}+\epsilon}^{2}} \\
& \leq c e^{-\epsilon j}\left\|v_{i}\right\|_{L_{\delta_{2}+\epsilon}^{2}} \\
& \leq c e^{-\epsilon(j-i)}\left\|v_{i}\right\|_{L_{\delta_{2}}^{2}}
\end{aligned}
$$

if we choose $\epsilon$ to be $\pm \epsilon$ according to the sign of $j-i$, we get the estimate

$$
\begin{aligned}
\left\|u_{i j}\right\|_{L_{\delta_{2}}^{2}} & \leq c e^{-\epsilon|i-j|}\left\|v_{i}\right\|_{L_{\delta_{2}}^{2}} \\
& \leq c e^{-\epsilon|i-j|}\left\|v_{i}\right\|_{L_{\delta}^{p}}
\end{aligned}
$$

now, note $\kappa_{i j}=1$ if $|i-j| \leq 1$ and 0 otherwise; using (11), we deduce

$$
\begin{aligned}
\left\|u_{i j}\right\|_{L_{\delta}^{p}} & \leq c\left(\kappa_{i j}\left\|v_{i}\right\|_{L_{\delta}^{p}}+e^{-\epsilon|i-j|}\left\|v_{i}\right\|_{L_{\delta_{2}}^{2}}\right) \\
& \leq c e^{-\epsilon|i-j|}\left\|v_{i}\right\|_{L_{\delta}^{p}}
\end{aligned}
$$

from which we deduce immediately

$$
\|u\|_{L_{\delta}^{p}} \leq c\|v\|_{L_{\delta}^{p}}
$$

which proves, with the help of local elliptic regularity, that the operator is an isomorphism $L_{\delta}^{2, p} \rightarrow L_{\delta}^{p}$.

Remark 2.4. Actually, the proof gives a bit more, namely the norm of the inverse operator is bounded by a constant which is independent of $R$ ( $R$ big enough); this is because we have explicit constants for the $L^{2}$ inverse, and the constants in the above proof do not depend on $R$.

Remark 2.5. The same proof works in Hölder spaces, and gives an isomorphism between Hölder weighted spaces. In $C^{k}$ spaces, we have no more elliptic regularity; nevertheless, if $v$ is in $C_{\delta}^{0}$, one can still deduce from the above proof the estimate

$$
\left\|r^{\delta-\epsilon} u\right\|_{C^{0}} \leq c\left\|r^{\delta} v\right\|_{C^{0}}
$$

this estimate is not a consequence of the $L^{p}$ estimate, because the Sobolev embedding (which can be proven like the elliptic estimate (11) by a homothety argument),

$$
\|u\|_{C_{\delta}^{0}} \leq c\left(\|u\|_{L_{\delta-2 / p}^{p}}+\|\nabla u\|_{L_{\delta+1-2 / p}^{p}}\right)
$$

implies $L_{\delta+1-2 / p}^{1, p} \hookrightarrow C_{\delta}^{0}$, so that there is a loss of weight, since $L_{\delta+1-2 / p}^{1, p}$ corresponds to functions $O\left(r^{-\delta-1}\right)$ when $C_{\delta}^{0}$ corresponds to functions $O\left(r^{-\delta}\right)$. Note also that in the case where $v$ lies in the component where $\gamma$ acts non trivially, the maximum principle provides directly the estimate (14) without the $\epsilon$. 
Analysis on 1-forms

In the next few lemmas, we assume that $a$ is a 1-form with values in $E n d(E)$ such that $\left.\partial_{r}\right\lrcorner a=0$ on $r=R$. Again we suppose that $a$ is reduced to its component $a_{\perp}$. All Sobolev norms are taken over the set $T \times V_{R}=\{r \geq R\}$.

Lemma 2.6. One has the identity

$$
\left.\left\|d_{\Gamma}^{*} a\right\|_{L^{2}}^{2}+\left\|d_{\Gamma} a\right\|_{L^{2}}^{2}=\left\|\nabla_{\Gamma} a\right\|_{L^{2}}^{2}-\int_{r=R} \mid \frac{1}{r} \frac{\partial}{\partial \theta}\right\lrcorner\left. a\right|^{2} d x d y d \theta
$$

with respect to the Euclidean metric.

Proof. The equality follows from the Weitzenböck formula in the Euclidean metric:

$$
d_{\Gamma}^{*} d_{\Gamma}+d_{\Gamma} d_{\Gamma}^{*}=\nabla_{\Gamma}^{*} \nabla_{\Gamma}
$$

Just integrate by parts and check the boundary terms.

Lemma 2.7. For any real function $f$ and any $R>0$, one has:

$$
f(R)^{2} \leq \frac{2}{R} \int_{R}^{R+1}\left(\left|\partial_{r} f\right|^{2}+|f|^{2}\right) r d r .
$$

The proof is left to the reader.

Lemma 2.8. If $R$ is sufficiently large, then for some constant c:

$$
\begin{gathered}
\left\|d_{\Gamma}^{*} a\right\|_{L_{\delta}^{p}}+\left\|d_{\Gamma} a\right\|_{L_{\delta}^{p}} \geq c\left\|\nabla_{\Gamma} a\right\|_{L_{\delta}^{p}} \\
\left\|d_{\Gamma}^{*} a\right\|_{C_{\delta}^{0}}+\left\|d_{\Gamma} a\right\|_{C_{\delta}^{0}} \geq c\|a\|_{C_{\delta-\epsilon}^{0}}
\end{gathered}
$$

with respect to the Euclidean metric.

Remark 2.9. Remind that on the component $a=a_{\perp}$ we look at, $\nabla_{\Gamma} a$ controls $a$ by (9).

Proof. From lemma 2.7 and lemma 2.1, we have:

$$
\begin{aligned}
\int_{r=R}|a|^{2} d x d y & \leq \frac{2}{R} \int_{[R, R+1]}\left(\left|\nabla_{\partial_{r}} a\right|^{2}+|a|^{2}\right) r d r d x d y \\
& \leq \frac{C_{1}}{R} \int_{[R, R+1]}\left|\nabla_{\Gamma} a\right|^{2} d x d y r d r
\end{aligned}
$$

for some constant $C_{1}$; in particular

$$
\left.\int_{r=R} \mid \frac{1}{r} \frac{\partial}{\partial \theta}\right\lrcorner\left. a\right|^{2} d x d y d \theta \leq \frac{C_{1}}{R} \int_{[R, R+1]}\left|\nabla_{\Gamma} a\right|^{2} d x d y r d r d \theta
$$

and we deduce from lemma 2.6, for $R$ big enough, 


$$
\left\|d_{\Gamma}^{*} a\right\|_{L^{2}}^{2}+\left\|d_{\Gamma} a\right\|_{L^{2}}^{2} \geq \frac{1}{2}\left\|\nabla_{\Gamma} a\right\|_{L^{2}}^{2}
$$

which proves the $L^{2}$-estimate of the lemma.

The $L^{2}$-estimate with weights is proven in the same way. In the integration by parts, new terms appear because of the weight $r^{2 \delta}$. However, as in the proof of lemma 2.2, these terms have all a coefficient $O\left(r^{-1}\right)$ and therefore are a small perturbation if $R$ is large enough (note that we can take the same $R$ if the weight remains bounded).

Finally, one may deduce the $L^{p}$ and $C^{0}$ estimates from the $L^{2}$ estimates as in lemma 2.3 and remark 2.5 , since the operator $d_{\Gamma}^{*}+d_{\Gamma}$ has injective symbol, and the boundary condition $\left.\partial_{r}\right\lrcorner a=0$ is an elliptic boundary condition. The proof is a slightly more complicated, because one has to compose the decomposition (13) with a $L^{2}$-projection on the image of the operator.

Lemma 2.10. The operator $2 d_{\Gamma}^{*} d_{\Gamma}^{+}+d_{\Gamma} d_{\Gamma}^{*}$ on 1 -forms lying in $\Omega^{1} \otimes \operatorname{End}(E)_{\perp}$, with Dirichlet condition on $r=R$, is an isomorphism in weighted Sobolev or Hölder spaces for all weights $\delta$.

Proof. Again the Weitzenböck formula

$$
2 d_{\Gamma}^{*} d_{\Gamma}^{+}+d_{\Gamma} d_{\Gamma}^{*}=\nabla_{\Gamma}^{*} \nabla_{\Gamma}
$$

gives the $L^{2}$-estimate (for forms vanishing on the boundary)

$$
\begin{aligned}
\left(\left(2 d_{\Gamma}^{*} d_{\Gamma}^{+}+d_{\Gamma} d_{\Gamma}^{*}\right) u, u\right)_{L^{2}} & =\left\|\nabla_{\Gamma} u\right\|_{L^{2}}^{2} \\
& \geq c\|u\|_{L^{2}}^{2}
\end{aligned}
$$

from which the $L^{2}$-statement (without weight) follows immediately. One can then deduce weighted statements as in the proofs of lemmas 2.2 and 2.3.

\section{Existence of a Coulomb gauge}

After the technical work of the previous section, we are finally in a position to establish theorem 0.6 , the key analytical result of this paper. The first step is the nonlinear version of the Hölder estimate in lemma 2.8; the exponent $p$ is fixed, near infinity.

Lemma 3.1. Given $\eta_{1}$ sufficiently small, if a connection $A=\Gamma+a_{\Gamma}+a_{\perp}$ on $r \geq R$ satisfies:

1. $d_{\Gamma+a_{\Gamma}}^{*} a_{\perp}=0$,

2. $\left.\partial_{r}\right\lrcorner a(r=R)=0$,

3. $\left\|r^{\epsilon} a\right\|_{C^{0}} \leq \eta_{1}$,

then:

$$
\left\|r^{2} F_{\Gamma+a_{\Gamma}}\right\|_{C^{0}}+\left\|r^{2-\epsilon} a_{\perp}\right\|_{C^{0}}+\left\|\left(\nabla_{\Gamma}+a_{\Gamma}\right) a_{\perp}\right\|_{L_{2-2 / p-\epsilon}^{p}} \leq c\left\|r^{2} F_{A}\right\|_{C^{0}} .
$$


Proof. First, note that:

$$
F_{A}=F_{\Gamma+a_{\Gamma}}+d_{\Gamma+a_{\Gamma}} a_{\perp}+\frac{1}{2}\left[a_{\perp}, a_{\perp}\right]
$$

Therefore, using the decomposition in (5), we have:

$$
\begin{aligned}
& \left(F_{A}\right)_{\Gamma}=F_{\Gamma+a_{\Gamma}}+\frac{1}{2}\left(\left[a_{\perp}, a_{\perp}\right]\right)_{\Gamma}, \\
& \left(F_{A}\right)_{\perp}=d_{\Gamma+a_{\Gamma}} a_{\perp}+\frac{1}{2}\left(\left[a_{\perp}, a_{\perp}\right]\right)_{\perp},
\end{aligned}
$$

from which the estimates below follow:

$$
\begin{aligned}
& \left\|r^{2}\left(F_{A}\right)_{\Gamma}\right\|_{C^{0}} \geq\left\|r^{2} F_{\Gamma+a_{\Gamma}}\right\|_{C^{0}}-\left\|r a_{\perp}\right\|_{C^{0}}^{2}, \\
& \left\|r^{2}\left(F_{A}\right)_{\perp}\right\|_{C^{0}} \geq\left\|r^{2} d_{\Gamma} a_{\perp}\right\|_{C^{0}}-\left\|r^{2}\left[a_{\Gamma}, a_{\perp}\right]\right\|_{C^{0}}-\left\|r a_{\perp}\right\|_{C^{0}}^{2} .
\end{aligned}
$$

Using $C_{2}^{0} \subset L_{2-2 / p-\epsilon}^{p}$ and the estimate in lemma 2.8, we get:

$$
\begin{gathered}
\left\|r^{2} F_{A}\right\|_{C^{0}} \geq c\left(\left\|r^{2} F_{\Gamma+a_{\Gamma}}\right\|_{C^{0}}+\left\|r^{2-\epsilon} a_{\perp}\right\|_{C^{0}}+\left\|\left(\nabla_{\Gamma}+a_{\Gamma}\right) a_{\perp}\right\|_{L_{2-2 / p-\epsilon}^{p}}\right) \\
-c^{\prime}\left(\left\|r a_{\perp}\right\|_{C^{0}}^{2}+\left\|r^{2}\left[a_{\Gamma}, a_{\perp}\right]\right\|_{C^{0}}\right) ;
\end{gathered}
$$

from the third hypothesis, we have

$$
\left\|r a_{\perp}\right\|_{C^{0}}^{2}+\left\|r^{2}\left[a_{\Gamma}, a_{\perp}\right]\right\|_{C^{0}} \leq \eta_{1}\left\|r^{2-\epsilon} a_{\perp}\right\|_{C^{0}} ;
$$

if $\eta_{1}$ is small enough, these two inequalities give the required estimate.

Lemma 3.2. Given $\eta$, there exists $R$ such that if $A$ is a connection over $T \times V_{R}$ such that $A-\Gamma$ is compactly supported and $\left|F_{A}\right| \leq \eta \cdot r^{-2}$, then there is a gauge g such that $g(A)=\nabla_{\Gamma}+a_{\Gamma}+a_{\perp}$, with:

(i) $d_{\Gamma+a_{\Gamma}}^{*} a_{\perp}=0$,

(ii) $\left.\partial_{r}\right\lrcorner a(r=R)=0$,

(iii) $\left\|r^{2} F_{\Gamma+a_{\Gamma}}\right\|_{C^{0}}+\left\|r^{2-\epsilon} a_{\perp}\right\|_{C^{0}}+\left\|\left(\nabla_{\Gamma}+a_{\Gamma}\right) a_{\perp}\right\|_{L_{2-2 / p-\epsilon}^{p}} \leq c\left\|r^{2} F_{A}\right\|_{C^{0}}$.

Proof. We now have all the necessary ingredients for a proof by continuity. Consider the homothety $\phi_{t}(r)=e^{t} r$ and the connections $A_{t}=\phi_{t}^{*} A$. We have $A_{0}=A$ and, for $t$ big enough, say $t \geq T, A_{t}=d_{\Gamma}$ because of the assumption on compact support. Moreover, it is clear from the form of the metric that

$$
\left|F_{A_{t}}\right|=\left|\phi_{t}^{*} F_{A}\right| \leq \phi_{t}^{*}\left|F_{A}\right| \leq \frac{c e^{-2 t}}{r^{2}}
$$

so that the whole path of connections $\left(A_{t}\right)$ satisfies the hypothesis of the lemma. Moreover, after gauge transformation, we can also assume that $A_{t}=\Gamma+a_{t}$ with $\left.\partial_{r}\right\lrcorner a_{t}(r=R)=0$ for all $t$. 
We prove that the subset $S \subseteq[0, T]$ containing all the values of $t$ for which the theorem holds for $A_{t}$ is both closed and open. Since $S$ is nonempty (it contains $t=T), S$ must be the whole interval and the result holds for $t=0$.

The closedness is trivial, since the estimate on the connection provides all the needed bounds.

For openness, first remark that proposition 1.5 provides a gauge in which

$$
\begin{aligned}
\left\|r^{2} F_{d_{\Gamma}+a_{\Gamma}}\right\|_{C^{0}} & \geq c\left\|\frac{r}{\ln r} a_{\Gamma}\right\|_{C^{0}} \\
& \geq c \frac{R^{1-\epsilon}}{\ln R}\left\|r^{\epsilon} a_{\Gamma}\right\|_{C^{0}} ;
\end{aligned}
$$

on the other hand, from (iii),

$$
\begin{aligned}
\left\|r^{2} F_{A}\right\|_{C^{0}} & \geq c\left\|r^{2-\epsilon} a_{\perp}\right\|_{C^{0}} \\
& \geq c R^{2-2 \epsilon}\left\|r^{\epsilon} a_{\perp}\right\|_{C^{0}}
\end{aligned}
$$

we deduce

$$
\left\|r^{\epsilon} a\right\|_{C^{0}} \leq c^{-1} R^{-(1-2 \epsilon)} \eta
$$

taking $R$ big enough, we deduce that (iii) implies

$$
\left\|r^{\epsilon} a\right\|_{C^{0}}<\frac{1}{2} \eta_{1}
$$

where $\eta_{1}$ comes from lemma 3.1. Of course, if (24) is true, then it remains true for any nearby $a$; this means that if we have a solution of (i), (ii) and (iii), then a nearby solution of (i) and (ii) again satisfies (24), so satisfies the hypothesis of lemma 3.1; applying this lemma, we deduce that the solution will also satisfy (iii). Therefore, the problem of openness for solutions of (i), (ii) and (iii) is reduced to solving problem (i)-(ii) near a solution.

Fix some $t$ and suppose that $g_{t}\left(A_{t}\right)=\Gamma+b$ with $\Gamma+b$ satisfying (i), (ii) and (iii). If we have a connection $\Gamma+b+\varpi$ with $\left.\partial_{r}\right\lrcorner \varpi(r=R)=0$, we want to find a gauge $g$ such that:

$$
\left\{\begin{array}{l}
g(\Gamma+b+\varpi)=\Gamma+c_{\Gamma}+c_{\perp} \\
d_{\Gamma+c_{\Gamma}}^{*} c_{\perp}=0 .
\end{array}\right.
$$

Looking at solutions of the form $g=e^{u_{\perp}}$, the equation to be solved is:

$$
L\left(u_{\perp}, \varpi\right)=d_{\Gamma+c_{\Gamma}}^{*}\left(e^{u}\left(\Gamma+c_{\Gamma}+c_{\perp}\right) e^{-u}-d_{\Gamma+c_{\Gamma}}\left(e^{u}\right) \cdot e^{-u}\right)=0 ;
$$

we would like to solve this equation with $u_{\perp}$ in $C^{2}$, but $C^{k}$ spaces are not suitable for elliptic analysis; instead, we use weighted $L^{p}$ spaces with $p$ very big; since we have the freedom to apply a $\Gamma$-invariant gauge transformation, using proposition 1.5 , we can choose a gauge in which the derivatives of $b_{\Gamma}$ are also controled, and therefore the operator $L$ is well defined; its linearization along the first variable is given by the operator:

$$
u \rightarrow d_{\Gamma}^{*} d_{\Gamma} u+\text { perturbation; }
$$

if $R$ is big enough, the perturbation is sufficiently small and we get an isomorphism by lemma 2.2 .

This completes the proof. 
Completing the proof of theorem 0.6. Our final task is to remove from lemma 3.2 the assumption that $A-\Gamma$ is compactly supported.

Using proposition 1.1, we approximate the connection $A$ by a sequence $A_{i}$ such that $\Gamma-A_{i}$ is compactly supported, and $\left\|r^{2} F_{A_{i}}\right\|_{C^{0}}$ remains bounded.

We can apply lemma 3.2 to each connection $A_{i}$, thus obtaining a gauge $g_{i}$ such that $g_{i}\left(A_{i}\right)=d_{\Gamma}+a_{i}$, and $a_{i}$ satisfies (i)-(iii) of lemma 3.2. Using proposition 1.5 for the $\left(a_{i}\right)_{\Gamma}$ part, the $\left(a_{i}\right)$ converge (weakly) to a limit $a$ still satisfying (i)-(iii), such that $d_{\Gamma}+a$ is gauge equivalent to $A$.

\section{Instantons, holomorphic bundles, and the moduli space}

So far, $A$ has simply been a connection on $E \rightarrow T \times \mathbb{C}$ with quadratic curvature decay. From now on, we shall assume that $A$ is also an instanton.

\section{Asymptotic behaviour: proof of theorem 0.1}

Let us now assume that $A$ is a doubly-periodic instanton connection. Using theorem 0.6, if $R$ is big enough, we can put it in a Coulomb gauge on $r \geq R$, so that $A=\Gamma+a_{\Gamma}+a_{\perp}$, with $a_{\Gamma}$ and $a_{\perp}$ satisfying the Coulomb gauge equation,

$$
d_{\Gamma+a_{\Gamma}}^{*} a_{\perp}=0,
$$

and the anti-self-duality equation,

$$
d_{\Gamma}^{+} a+\frac{1}{2}[a, a]^{+}=0 .
$$

These can be rewritten as follows:

$$
\begin{aligned}
d_{\Gamma}^{*} a_{\perp} & =-a_{\Gamma}^{*} a_{\perp} \\
d_{\Gamma}^{+} a_{\perp} & =-\left[a_{\Gamma}, a_{\perp}\right]^{+}-\frac{1}{2}\left[a_{\perp}, a_{\perp}\right]_{\perp}^{+} \\
d^{+} a_{\Gamma}+\frac{1}{2}\left[a_{\Gamma}, a_{\Gamma}\right]^{+} & =-\left[a_{\perp}, a_{\perp}\right]_{\Gamma}^{+}
\end{aligned}
$$

Now let $\chi=\chi(r)$ be a smooth cut-off function supported on $T \times V_{R}$; we have, using equations (26) and (27):

$$
\left(d_{\Gamma}^{+}+d_{\Gamma}^{*}\right)\left(\chi a_{\perp}\right)=\chi\left(a_{\Gamma} \odot a_{\perp}+a_{\perp} \odot a_{\perp}\right)+d \chi \odot a_{\perp}
$$

where $\odot$ denotes some bilinear operations.

From theorem 0.6 and proposition 1.5 , we already know that $\left|a_{\perp}\right|=O\left(r^{-2+\epsilon}\right)$ and that we can choose a gauge such that $\left|a_{\Gamma}\right|=O(\ln r / r)$. We now apply lemma 2.10 to the equation (28): a priori the lemma applies to the Laplacian $\left(d_{\Gamma}^{+}\right)^{*} d_{\Gamma}^{+}+d_{\Gamma} d_{\Gamma}^{*}$ but the estimates also imply estimates for the first order elliptic operator $d_{\Gamma}^{+}+d_{\Gamma}^{*}$ (alternatively one may take one derivative of equation (28) and use the bounds on the derivatives of $a_{\perp}$ and $a_{\Gamma}$ ); the RHS of equation (28) is 
$O\left(r^{-3+\epsilon}\right)$, therefore $\left|a_{\perp}\right|=O\left(r^{-3+\epsilon_{2}}\right)$, where $\epsilon_{2}>\epsilon$; by the same argument, we have that $\left|a_{\perp}\right|=O\left(r^{-4+\epsilon_{3}}\right)$, etc. Therefore, $\left|a_{\perp}\right|=O\left(r^{-\delta}\right)$ for any $\delta>0$.

Now come back to equation (25): it now means that $d+a_{\Gamma}$ satisfies the instanton equation up to a term which goes very quickly to 0 at infinity; as $a_{\Gamma}$ is translation invariant, this means, by dimensional reduction, that $d+a_{\Gamma}$ is a solution of Hitchin's equations for Higgs bundles on $\mathbb{R}^{2}$ near infinity, up to a term decaying quicker than any $O\left(r^{-\delta}\right)$. The behaviour of the solutions of Hitchin's equations near a singularity has been studied by Simpson [22], Biquard [3]. The arguments in these papers are not affected by a very quickly decaying perturbation. Moreover, the bounds in proposition 1.5 implies that the Higgs field is $O(1 / r)$ at infinity, so that the Higgs bundle is "tame" in Simpson's terminology. Finally, we deduce from these articles that $d+a_{\Gamma}$ is close to one of the examples described in the introduction, in the sense of theorem 0.1 .

\section{Holomorphic extension}

The theorem 0.1 proves that any instanton $A$ with quadratic curvature decay can be put in a gauge near infinity so that

$$
A=A_{0}+a,
$$

where $A_{0}$ is one of the model torus invariant instantons induced by model Higgs bundles, and $a$ is a small perturbation.

Local aspects

Let us now restrict to the semisimple case. Therefore, we have

$$
A_{0}=d+i\left(\begin{array}{cc}
a_{0} & 0 \\
0 & -a_{0}
\end{array}\right)
$$

with

$$
a_{0}=\lambda_{1} d x+\lambda_{2} d y+\left(\mu_{1} \cos \theta-\mu_{2} \sin \theta\right) \frac{d x}{r}+\left(\mu_{1} \sin \theta+\mu_{2} \cos \theta\right) \frac{d y}{r}+\alpha d \theta ;
$$

observe that the $(0,1)$-part of this form is

$$
a_{0}^{0,1}=\lambda d \bar{z}+\mu \frac{d \bar{z}}{w}-\frac{\alpha}{2} \frac{d \bar{w}}{\bar{w}}, \quad \lambda=\frac{\lambda_{1}+i \lambda_{2}}{2}, \mu=\frac{\mu_{1}+i \mu_{2}}{2},
$$

so there is a singularity in the direction of transverse disks to the torus at infinity. We first reduce to a normal form on transverse disks.

Lemma 5.1. Near the torus at infinity, there exists a continuous complex gauge transformation $g$, such that

1. $\left.g\right|_{T_{\infty}}=1$;

2. $\left|\nabla_{A_{0}} g g^{-1}\right|=O\left(r^{-(1+\delta)}\right)$ (and $g_{\perp}$ is $O\left(r^{-\delta}\right)$ for any $\left.\delta\right)$;

3. $g\left(\bar{\partial}_{A}\right)=\bar{\partial}_{A_{0}}+b d \bar{z}$, with $b=O\left(r^{-(1+\delta)}\right)$. 
Proof. We give a concise proof, since this is parallel to [3, Sect. 9]. Remark that

$$
\bar{\partial}_{\alpha}=\bar{\partial}-\frac{\alpha}{2} \frac{d \bar{w}}{\bar{w}}=r^{-\alpha} \circ \bar{\partial} \circ r^{\alpha} ;
$$

now the problem to be solved is

$$
\frac{\bar{\partial}_{A_{0}}}{\partial \bar{w}} g-g a=0
$$

that is, using $g=1+u$,

$$
\left(\frac{\partial}{\partial \bar{w}}-\frac{1}{2}\left(\begin{array}{cc}
\alpha & 0 \\
0 & -\alpha
\end{array}\right)\right) u-u a=-a ;
$$

this is a $\bar{\partial}$-problem on small disks near infinity; for the model problem (29) the Cauchy formula gives us an explicit solution; in general, with the small perturbation $a$, the solution is produced by a fixed point theorem, and we even have an estimate

$$
\sup r^{\delta}|u| \leq c \sup r^{1+\delta}|a| ;
$$

one can then deduce the regularity statement on $u$.

Note $b_{j k}$ the coefficients of the matrix $b$ above. Let $\left(e_{1}, e_{2}\right)$ be the orthonormal basis for the trivialisation of the bundle near infinity. From the lemma and equation (29), we deduce that the sections

$$
\left(\sigma_{1}=r^{-\alpha} g\left(e_{1}\right), \sigma_{2}=r^{\alpha} g\left(e_{2}\right)\right)
$$

are holomorphic on transverse disks, and, moreover, in the basis $\left(\sigma_{1}, \sigma_{2}\right)$, we now have

$$
\bar{\partial}_{A}=\bar{\partial}+\left(\begin{array}{cc}
\lambda & 0 \\
0 & -\lambda
\end{array}\right) d \bar{z}+\left(\begin{array}{cc}
\mu & 0 \\
0 & -\mu
\end{array}\right) \frac{d \bar{z}}{w}+\left(\begin{array}{cc}
b_{11} & r^{2 \alpha} b_{12} \\
r^{-2 \alpha} b_{21} & b_{22}
\end{array}\right) d \bar{z}
$$

with all coefficients of the last matrix holomorphic in $w$. From this, we see immediately that in the basis $\left(\sigma_{1}, \sigma_{2}\right)$, the operator (31) defines a holomorphic extension $\mathcal{E}$ over $T \times \mathbb{P}^{1}$.

Since

$$
\left|\sigma_{1}\right| \sim r^{-\alpha}, \quad\left|\sigma_{2}\right| \sim r^{\alpha},
$$

we see that, from an intrinsic point of view, if $\alpha<1 / 2$, the local holomorphic sections of $\mathcal{E}$ are characterized as the local holomorphic sections $\sigma$ outside $T_{\infty}$ satisfying the growth condition

$$
|\sigma|=O\left(r^{\alpha}\right)
$$

When $0<\alpha<1 / 2$, this global extension has a subbundle $\mathcal{F}$ over the torus at infinity, given by the values of the local holomorphic sections $\sigma$ satisfying the growth condition

$$
|\sigma|=O\left(r^{-\alpha}\right)
$$


Therefore, the growth of the holomorphic sections at infinity determine a "parabolic structure"

$$
\mathcal{E} \supset \mathcal{F} \supset 0
$$

with weights $-\alpha<\alpha$ (the sign is changed because the local coordinate near infinity is $w^{-1}$ ).

Actually one can say more: over $T_{\infty}$, the $\bar{\partial}$-operator (31) is

$$
\bar{\partial}+\left(\begin{array}{cc}
\lambda & 0 \\
0 & -\lambda
\end{array}\right) d \bar{z}
$$

which means that

$$
\left.\mathcal{E}\right|_{T_{\infty}}=L_{\xi_{0}} \oplus L_{-\xi_{0}} .
$$

Of course, if $\alpha$ is nontrivial, then $\mathcal{F}=L_{\xi_{0}}$ is canonically determined by the growth condition (33).

Actually, the decomposition $L_{\xi_{0}} \oplus L_{-\xi_{0}}$ can almost always be made canonical: this is clear if $\xi_{0} \neq 0$, and in this case, since the off-diagonal components of the connection decay quicker than any $O\left(r^{-\delta}\right)$, we deduce from equation (31) that, still in the basis $\left(\sigma_{1}, \sigma_{2}\right)$,

$$
\bar{\partial}_{A}=\bar{\partial}+\left(\begin{array}{cc}
\lambda & 0 \\
0 & -\lambda
\end{array}\right) d \bar{z}+\left(\begin{array}{cc}
\mu & 0 \\
0 & -\mu
\end{array}\right) \frac{d \bar{z}}{w}+O\left(r^{-2}\right) ;
$$

this gives the asymptotic behaviour of $\left.\mathcal{E}\right|_{T_{w}}$ when $w$ goes to infinity.

Moreover, when $\xi_{0}=0$, we still get something from (31): since the coefficients are holomorphic in $w$, we note $b_{12}^{\prime}$ the coefficient of $r^{2 \alpha} b_{12}$ on $w^{-1}$ (in the case $\alpha=0$, we simply have $b_{12}^{\prime}=0$ ), so

$$
\bar{\partial}_{A}=\bar{\partial}+\left(\begin{array}{cc}
\mu & b_{12}^{\prime} \\
0 & -\mu
\end{array}\right) \frac{d \bar{z}}{w}+O\left(r^{-2}\right)
$$

if $\mu \neq 0$, the matrix appearing above can always been diagonalized with eigenvalues $\pm \mu$, which means that up to changing $\sigma_{2}$ by some multiple of $\sigma_{1}$, we are reduced to (34) so that a supplementary subspace of $\mathcal{F}$ is still well defined (and when $\alpha=0$, the decomposition $\mathbb{C} \oplus \mathbb{C}$ still makes sense, as the eigenspaces of this matrix).

Note also that, as a consequence of (30), since $g$ is continuous, the unitary extension (given by the basis $\left(e_{1}, e_{2}\right)$ of the Coulomb gauge) and the holomorphic extension are topologically isomorphic.

Therefore, we have proven the following proposition.

Proposition 5.2. In the semisimple case, for $\alpha<1 / 2$, if $A$ is a doubly-periodic instanton connection satisfying $\left|F_{A}\right|=O\left(r^{-2}\right)$, then $A^{0,1}$ has a unique holomorphic extension $\mathcal{E}$ over $T \times \mathbb{P}^{1}$, whose holomorphic sections satisfy the growth condition (32). Moreover, one has $c_{2}(\mathcal{E})=k$ and a decomposition (if $\lambda$ or $\mu$ is nonzero) $\left.\mathcal{E}\right|_{T_{\infty}}=L_{\xi_{0}} \oplus L_{-\xi_{0}}$. 
Remark 5.3. Note that when $\alpha=1 / 2$, we cannot get a $S l_{2}$-extension this way: indeed we could equally well choose the sections $\left(w \sigma_{1}, \sigma_{2} / w\right)$, giving a different extension. One way to construct a canonical extension is to use (32) with $\alpha=-1 / 2$, which furnishes a $G l_{2}$-extension where all nonzero sections have norm $O\left(r^{-1 / 2}\right)$. Also, a $S l_{2}$-extension can be constructed if $\xi_{0} \neq-\xi_{0}$, by deciding that sections with nonzero values in $L_{ \pm \xi_{0}}$ have norm like $r^{\mp 1 / 2}$.

In the sequel we will ignore this case, but all the statements can be easily adapted to it.

Remark 5.4. In the nilpotent case (then $\lambda, \mu$, and $\alpha$ are trivial), the result is the same, but (as in the case of Higgs bundles) the growth of the holomorphic sections at infinity is now logarithmic:

$$
|\sigma|=O\left((\ln r)^{\frac{1}{2}}\right)
$$

and there is a line subbundle $\mathcal{F}$ defined by the growth condition

$$
|\sigma|=O\left((\ln r)^{-\frac{1}{2}}\right)
$$

The subbundle $\mathcal{F}$ has no canonical supplementary subspace. The tools in [3, Sect. 9] handle this situation as well.

Also observe that the $\bar{\partial}$-operator for the model instanton (3) is (in an orthonormal basis $\left.\left(e_{1}, e_{2}\right)\right)$

$$
\bar{\partial}+\left(\begin{array}{cc}
1 & 0 \\
0 & -1
\end{array}\right) \frac{d \bar{w}}{2 \bar{w} \ln r^{2}}+\frac{1}{r \ln r^{2}}\left(\begin{array}{cc}
0 & e^{-i \theta} d \bar{z} \\
0 & 0
\end{array}\right)
$$

which gives, in the basis $\left(e_{1} /\left(\ln r^{2}\right)^{\frac{1}{2}}, e_{2}\left(\ln r^{2}\right)^{\frac{1}{2}}\right)$,

$$
\bar{\partial}+\left(\begin{array}{cc}
0 & \frac{d \bar{z}}{w} \\
0 & 0
\end{array}\right)
$$

in particular, $\left.\mathcal{E}\right|_{T_{w}}$ is the nontrivial extension of $\underline{\mathbb{C}}$ by $\underline{\mathbb{C}}$; it is easy to see that this remains true for instantons, asymptotic to this nilpotent model.

Non-existence results

The proposition 5.2 gives obstructions for the existence of instantons. Here are some examples.

Lemma 5.5. There are no instantons with $\xi_{0}=-\xi_{0}$ and $k=1$.

Proof. For a contradiction, let $A$ be an instanton with $\xi_{0}=-\xi_{0}$ and $k=1,2$, and consider the extended holomorphic bundle $\mathcal{E}$ given by theorem 0.2 . The restriction of $\mathcal{E}$ to the elliptic fibres $T_{p}$ must be semistable for all $p \in \mathbb{P}^{1}$ (see [14]). Moreover, $\left.\mathcal{E}\right|_{T_{p}}$ cannot be generically the nontrivial extension of $\mathbb{C}$ by itself, since this would give a non-constant map from $\mathbb{P}^{1}$ to $\mathbb{C}$ (which parametrises the extensions of $\underline{\mathbb{C}}$ by itself). 
Therefore, as shown in $[13,14]$, index theory tells us that for each $\xi \in \hat{T}$ :

$$
\Sigma_{w \in \mathbb{P}^{1}} h^{0}\left(T_{w},\left.\mathcal{E} \otimes L_{\xi}\right|_{T_{w}}\right)=k .
$$

But if $\left.\mathcal{E}\right|_{T_{\infty}}=L_{\xi_{0}} \oplus L_{\xi_{0}}$, then $h^{0}\left(T_{\infty},\left.\mathcal{E} \otimes L_{\xi_{0}}\right|_{T_{\infty}}\right)=2$, thus contradicting the assumption that $k=1$.

Lemma 5.6. There are no instantons with $\xi_{0} \neq-\xi_{0}$ and $\mu=0$.

Proof. The lemma is a consequence of the Nahm transform of doubly-periodic instantons defined in [13], more exactly of its holomorphic aspects; we anticipate a bit here, but see the introduction to Part III for a summary of the construction.

Again for a contradiction, let $A$ be an instanton with $\mu=0$ and asymptotic state $\pm \xi_{0}$ not of order two. The corresponding Nahm transformed Higgs field $\Phi$ has simple poles at $\pm \xi_{0}$; its residues have rank one. However, as we shall see in the proof of theorem 0.4 , the non-zero eigenvalues of the residues of $\Phi$ are exactly $\pm \mu$, and more generally, the eigenvalues of $\Phi$ at $\xi \in \hat{T}$ are the $w$ such that $H^{0}\left(T_{w}, \mathcal{E} \otimes L_{\xi}\right) \neq 0$; hence, the vanishing of $\mu$ implies that the eigenvalues of $\Phi$ remain bounded when $\xi$ goes to $\xi_{0}$.

Now if $\xi_{0} \neq \xi_{0}$ then $\mathcal{E}$ remains isomorphic to some $L_{\xi} \oplus L_{-\xi}$ on each torus near infinity. It is then clear (again, see the proof of theorem 0.4 ) that the eigenvalues of $\Phi$ must go to infinity and we get a contradiction.

Global aspects, stability

More subtle obstructions come from stability properties. We investigate this for the extension $\mathcal{E}$ of an instanton $A$ with quadratic curvature decay. Notice that by theorem 0.1 , in the semisimple case, the curvature is only $O\left(r^{-2}\right)$, but

$$
\left|\iota_{\{\cdot\} \times \mathbb{C}} F_{A}\right|+\left|\iota_{T \times\{\cdot\}} F_{A}\right|=O\left(r^{-(2+\epsilon)}\right) ;
$$

in the nilpotent case, we have

$$
\left|F_{A}\right|=O\left(r^{-2}(\ln r)^{-2}\right)
$$

the point here is that these two controlling factors are in $L^{1}$, whence $F_{A}$ itself is not $L^{1}$ : this will enable us to define a degree.

The degree of a saturated subsheaf $L$ of $\mathcal{E}$ with respect to the Euclidean Kähler form $\omega$ is [21, lemma 3.2]

$$
2 \pi \operatorname{deg} L=i \int \operatorname{tr}\left(\pi F_{A}\right) \wedge \omega-\int|\bar{\partial} \pi|^{2}
$$

where $\pi$ is orthogonal projection on $L$; from (39) and (40), this can be $-\infty$ or a real number; in the last case, $\bar{\partial} \pi$ is in $L^{2}$ : this condition must be analyzed more precisely.

Again, we now restrict to the semisimple case (see remark 5.10 for the nilpotent case), so that $\left.\mathcal{E}\right|_{T_{\infty}}=L_{\xi_{0}} \oplus L_{-\xi_{0}}$, with weights $-\alpha$ and $\alpha$, and behaviour (34). In this case, we have near infinity

$$
\left.\mathcal{E}\right|_{T_{w}}=L_{\xi(w)} \oplus L_{-\xi(w)} .
$$


Lemma 5.7. Suppose $\alpha \neq 0$, then the degree of a subsheaf $L$ of $\mathcal{E}$ is finite if and only if

1. $\left.L\right|_{T_{\infty}}$ is flat; in particular, if $L_{\xi_{0}} \neq L_{-\xi_{0}}$, this means that $L \subset L_{ \pm \xi_{0}}$;

2. if $\left.L\right|_{T_{\infty}} \subset \mathcal{F}=L_{\xi_{0}}$, then $\left.L\right|_{T_{w}} \subset L_{\xi(w)}$ up to first order near infinity.

Now suppose $\alpha=0$, then the degree of a subsheaf $L$ of $\mathcal{E}$ is finite if and only if $\left.L\right|_{T_{w}} \subset L_{ \pm \xi(w)}$ up to first order near infinity.

Remark 5.8. The first order condition can be seen as a reminiscence of the approximating Higgs bundle at infinity; indeed the Higgs field has eigenspaces $L_{ \pm \xi(w)}$ and for Higgs bundle stability, one looks only at subsheaves stable under the action of the Higgs field.

Proof. We analyze the situation locally near infinity; in the decomposition (42), the metric is approximately

$$
\left(\begin{array}{cc}
r^{-2 \alpha} & 0 \\
0 & r^{2 \alpha}
\end{array}\right)
$$

and we will simplify the problem by using this metric to make the calculations (the correction term can be easily bounded); at a point on $T_{\infty}$ where $L$ is a subbundle, we suppose for example that $L$ is not contained in $L_{-\xi_{0}}$; choose a local flat section $\sigma$ for $L_{\xi_{0}}$, and note $\sigma^{t}$ the dual flat section of $L_{-\xi_{0}}$; extend $\sigma$ near $T_{\infty}$, keeping it parallel on $T_{w}$ (this is possible with our approximation for the metric); locally, $L$ is generated by $s=\sigma+f \sigma^{t}$, where $f$ is holomorphic, and an orthogonal section is given by $t=r^{2 \alpha} \sigma-\bar{f} \sigma^{t} r^{-2 \alpha}$, and

$$
\bar{\partial}_{T} t=-\left(\bar{\partial}_{T} \bar{f}\right) \sigma^{t} r^{-2 \alpha}
$$

from which we deduce

$$
\pi\left(\bar{\partial}_{T} t\right)=-\frac{\bar{\partial}_{T} \bar{f}}{r^{-2 \alpha}+|f|^{2} r^{2 \alpha}} s,
$$

and finally, since our choice of $t$ satisfies $|s|=|t|$, and $f$ is holomorphic,

$$
\left|\bar{\partial}_{T} \pi\right|=\frac{\left|d_{T} f\right|}{r^{-2 \alpha}+|f|^{2} r^{2 \alpha}}
$$

in order for $\bar{\partial}_{T} \pi$ to be in $L^{2}$, it is necessary that $d_{T} f=0$ on $T_{\infty}$, and therefore $L$ is constant.

Now restrict to the case of nontrivial decomposition $L_{\xi_{0}} \oplus L_{-\xi_{0}}$ (the other cases are similar); therefore we may suppose that $f=0$ on $T_{\infty}$; if the first order term of $d_{T} f$ does not vanish, then

$$
\left|\bar{\partial}_{T} \pi\right| \sim r^{-1+2 \alpha}
$$

this still is not in $L^{2}$ if $\alpha \geq 0$ (but it is in $L^{2}$ if $\alpha<0$, which corresponds to the case $\left.L\right|_{T_{\infty}} \subset L_{-\xi_{0}}$ ); this means that we need $d_{T} f$ to vanish up to first order.

Concerning $\bar{\partial}_{\mathbb{C}} \pi$, it is easy to verify that the $L^{2}$-condition is always satisfied. 
Recall that

$$
F_{L}=\pi F_{A} \pi+\bar{\partial} \pi \wedge \partial \pi .
$$

When the degree is finite, that is when $\bar{\partial} \pi$ is $L^{2}$, the restriction of $\omega$ to $\mathbb{C}$ does not contribute: indeed, $d w \wedge d \bar{w}=\partial \bar{\partial}|w|^{2}$, and this leads to

$$
\int_{r \leq R} F_{L} \wedge d w \wedge d \bar{w}=\int_{r=R} w d w \wedge F_{L}
$$

but using (39) and (43), we see that this goes to zero as $R$ goes to infinity. Then we can rewrite the degree (denoting $\bar{\partial}_{\mathbb{C}}$ the $\bar{\partial}$ operator in the $\mathbb{C}$ direction)

$$
2 \pi \operatorname{deg} L=i \int \pi F_{A} \wedge \omega_{T}-\int\left|\bar{\partial}_{\mathbb{C}} \pi\right|^{2},
$$

and this in turn is easily interpreted [2, (4.1)] as a "parabolic degree":

$$
\operatorname{deg} L= \begin{cases}c_{1}(L)[t]+\alpha\left\langle\left[\omega_{T}\right],[t]\right\rangle & \text { if } L_{T_{\infty}} \subset L_{-\xi_{0}}, \\ c_{1}(L)[t]-\alpha\left\langle\left[\omega_{T}\right],[t]\right\rangle & \text { if } L_{T_{\infty}} \subset L_{\xi_{0}},\end{cases}
$$

where $[t]$ is the fundamental class of $T$ and $\omega_{T}$ the given Kähler form on $T$; of course this is not a degree in the usual sense on $T \times \mathbb{P}^{1}$, since we use the non ample class $[t]$.

Define $\alpha$-stability of $\mathcal{E}$ as the fact that any subsheaf satisfying the condition of lemma 5.7 has negative degree (we shall forget the $\alpha$ when there is no ambiguity); standard arguments give us

Proposition 5.9. If $A$ is an instanton with quadratic curvature decay, then the holomorphic extension $\mathcal{E}$ is $\alpha$-stable.

Remark 5.10. In the nilpotent case, the proposition remains true; here $\alpha=0$, and, following the proof of lemma 5.7, the degree is finite for all subsheaves with flat restriction to $T_{\infty}$.

Remark 5.11. It is important to note that the stability condition just defined is not an empty one. Indeed, $\alpha$-unstable bundles $\mathcal{E} \rightarrow T \times \mathbb{P}^{1}$ can be obtained as extensions in the following way:

$$
0 \rightarrow p_{1}^{*} L_{\xi_{0}} \otimes p_{2}^{*} \mathcal{O}_{\mathbb{P}^{1}}(b) \rightarrow \mathcal{E} \rightarrow p_{1}^{*} L_{-\xi_{0}} \otimes p_{2}^{*} \mathcal{O}_{\mathbb{P}^{1}}(-b) \otimes \mathcal{I}_{k} \rightarrow 0
$$

where $b>0$ and $\mathcal{I}_{k}$ is the ideal sheaf of $k>0$ points in $T \times \mathbb{P}^{1}$, and we assume that none of these points are in $T_{\infty}$. Every sheaf $\mathcal{E}$ so obtained is locally-free, since the sheaf on the LHS is locally-free and the one on the RHS is torsion-free. Clearly, $\mathcal{E}$ has trivial determinant, instanton number $k$ and asymptotic states $\pm \xi_{0}$.

To finish the proof of theorem 0.2 , it remains to prove the following proposition.

Proposition 5.12. Every $\alpha$-stable, holomorphic Sl$\ell_{2}$-bundle $\mathcal{E}$ over $T \times \mathbb{P}^{1}$ restricting to $L_{\xi_{0}} \oplus L_{-\xi_{0}}$ on $T_{\infty}$ can be obtained as the holomorphic extension of an instanton on $T \times \mathbb{C}$ with asymptotic states $\pm \xi_{0}$, and whose monodromy around the torus at infinity has eigenvalues $\exp ( \pm 2 \pi i \alpha)$. 
Proof. We will give only the idea to prove the proposition, but we will not give the proof, because it essentially follows well known arguments.

The idea consists in constructing a Hermitian-Einstein metric on $\left.\mathcal{E}\right|_{T \times \mathbb{C}}$ (so that the Chern connection is anti-self-dual); for this, one has first to build a metric $h_{0}$ on $\mathcal{E}$ which gives asymptotically at infinity an instanton: this is possible because $\alpha$ and the behaviour of $\mathcal{E}$ near infinity (see (34)) give all the parameters at infinity of the instanton; then one wants to deform $h_{0}$ to a solution $h$ of the Hermitian-Einstein equation, mutually bounded with $h_{0}$; Simpson's method by exhaustion [21] cannot be used, because $T \times \mathbb{C}$ has infinite volume; fortunately, the method in [3] of direct minimization of Donaldson's functional in suitable functional spaces can handle this kind of situation, provided that some precise analysis at infinity is developed; this is precisely done in the next section for the study of the moduli space.

Remark 5.13. There is probably another possible proof, which requires some delicate algebraic geometry that we will not discuss here: it consists in reducing to the above mentioned theorem of Simpson throw the Nahm transform of instantons. Recall that our instantons are in correspondence with Higgs bundles with singularities on the dual torus $\hat{T}$, with a harmonic metric. Actually, the correspondence has a purely holomorphic interpretation, and this is an occurrence of the so-called Fourier-Mukai transform. It is known [8] that the Fourier-Mukai transform on abelian surfaces preserves stability in the case $c_{1}=0$, so one may hope to prove a similar statement in our case (we also have $c_{1}=0$, since we consider $\mathrm{S}_{2}$-bundles); if this is true, an $\alpha$-stable bundle on $T \times \mathbb{P}^{1}$ will transform into a stable parabolic Higgs bundle on $\hat{T}$; then one can apply Simpson's theorem [22] to construct a harmonic metric, whose inverse Nahm transform provides an instanton with quadratic curvature decay, and by theorem 0.1 this instanton has exactly the desired behaviour at infinity.

\section{Moduli spaces}

We now proceed to the differential geometric construction of the moduli space. The $L^{2}$ metric will then provide a hyperkähler structure on it.

We will restrict to the semisimple case; this choice simplifies the construction, because theorem 0.1 says that it is enough to look at functional spaces with weights which are powers of $r$; the analysis in the nilpotent case is possible, as in [3], but requires functional spaces with logarithmic weights.

Recall the model connection on the bundle $E$, trivialized near infinity:

$$
\begin{aligned}
A_{0}=d & +i\left(\begin{array}{cc}
\lambda_{1} & 0 \\
0 & -\lambda_{1}
\end{array}\right) d x+i\left(\begin{array}{cc}
\lambda_{2} & 0 \\
0 & -\lambda_{2}
\end{array}\right) d y \\
& +i\left(\begin{array}{cc}
\mu_{1} \cos \theta-\mu_{2} \sin \theta & 0 \\
0 & -\mu_{1} \cos \theta+\mu_{2} \sin \theta
\end{array}\right) \frac{d x}{r} \\
& +i\left(\begin{array}{cc}
\mu_{1} \sin \theta+\mu_{2} \cos \theta & 0 \\
0 & -\mu_{1} \sin \theta-\mu_{2} \cos \theta
\end{array}\right) \frac{d y}{r}+ \\
& +i\left(\begin{array}{cc}
\alpha & 0 \\
0 & -\alpha
\end{array}\right) d \theta .
\end{aligned}
$$


Note that in order to get $L^{2}$ deformations, we cannot move the parameters $\lambda, \mu$ and $\alpha$; in view of theorem 0.1 , it is natural to consider connections $A_{0}+a$, such that

$$
|a|=O\left(r^{-(1+\delta)}\right), \quad\left|\nabla_{A_{0}} a\right|=O\left(r^{-(2+\delta)}\right)
$$

actually, this $C^{1}$ space is not good for analysis, and we have the choice to substitute either a Hölder space $C^{1, \eta}$ or a Sobolev space $L^{1, p}$; we make the last choice, for $p$ big enough, and this leads to the technical definitions

$$
\begin{aligned}
\Omega_{\delta}^{1} & =\left\{a \in \Omega^{1}(\mathfrak{s u}(E)), a \in L_{1-2 / p+\delta}^{p}, \nabla_{A_{0}} a \in L_{2-2 / p+\delta}^{p}\right\} \\
\mathcal{A} & =A_{0}+\Omega_{\delta}^{1} \\
\mathcal{G} & =\left\{g \in S U(E), \nabla_{A_{0}} g g^{-1} \in \Omega_{\delta}^{1}\right\} \\
\mathcal{F} & =\left\{F \in \Omega_{+}^{2}(\mathfrak{s u}(E)), F \in L_{2-2 / p+\delta}^{p}\right\} .
\end{aligned}
$$

The Lie algebra of $\mathcal{G}$ is

$$
T_{1} \mathcal{G}=\left\{u \in \mathfrak{s u}(E), \nabla_{A_{0}} u \in \Omega_{\delta}^{1}\right\}
$$

Note that for $a \in \Omega_{\delta}^{1}$, lemma 2.1 implies that actually $a_{\perp} \in L_{2-2 / p+\delta}^{p}$, so that this Sobolev space is the same as the one considered is part I. Also, the Sobolev embedding (15) implies $\Omega_{\delta}^{1} \subset C_{\delta}^{0}$, and an important property is that the embedding $\Omega_{\delta}^{1} \subset C_{\delta^{\prime}}^{0}$ is compact if $\delta^{\prime}<\delta$; gauge transformations $g \in \mathcal{G}$ can be continuously extended over $T_{\infty}$, so that

$$
\left.g\right|_{T_{\infty}}=\left(\begin{array}{cc}
u & 0 \\
0 & u^{-1}
\end{array}\right),
$$

where $u \in S^{1}$ is fixed. Also, $\mathcal{G}$ acts smoothly on $\mathcal{A}$ and the curvature is a smooth map from $\mathcal{A}$ to $\mathcal{F}$.

Remark that there is no reducible connection in $\mathcal{A}$, since a reduction would decompose the bundle $E$ as $L \oplus L^{-1}$, with $L$ topologically trivial on the torus at infinity; but then we would get $c_{2}(E)=0$.

Now we need the following proposition; the proof is given at the end of the section.

Proposition 6.1. For $k>0$ and $A \in \mathcal{A}$, we have:

1. the Laplacian $\Delta_{A}: T_{1} \mathcal{G} \rightarrow L_{2-2 / p+\delta}^{p}$ is an isomorphism; therefore there is a slice at $A$ to the action of $\mathcal{G}$ on $\mathcal{A}$, given by $\left\{A+a, d_{A}^{*} a=0\right\}$;

2. if $A$ is an instanton, then the map $d_{A}^{+} \oplus d_{A}^{*}: \Omega_{\delta}^{1} \rightarrow L_{2-2 / p+\delta}^{p}$ is Fredholm surjective; the kernel coincide with the $L^{2}$-kernel.

Note that in the first statement of the proposition, it was crucial to allow gauge transformations to take non trivial values on $T_{\infty}$, otherwise one cannot obtain the slice $d_{A}^{*} a=0$. 
Define the moduli space $\mathcal{M}$ as the space of instantons $A \in \mathcal{A}$ modulo the gauge group $\mathcal{G}$. As is well-known, $F_{A}^{+}$is a hyperkähler moment map for the action of $\mathcal{G}$ on $\mathcal{A}$ with respect to the three complex structures on $T \times \mathbb{C}$ :

$$
\begin{aligned}
& I_{1}\left(z_{1}, z_{2}, w_{1}, w_{2}\right)=\left(-z_{2}, z_{1},-w_{2}, w_{1}\right) \\
& I_{2}\left(z_{1}, z_{2}, w_{1}, w_{2}\right)=\left(-w_{1}, w_{2}, z_{1},-z_{2}\right) \\
& I_{3}\left(z_{1}, z_{2}, w_{1}, w_{2}\right)=\left(-w_{2},-w_{1}, z_{2}, z_{1}\right)
\end{aligned}
$$

where $z=z_{1}+i z_{2}$ and $w=w_{1}+i w_{2}$. With the help of the previous proposition, standard theory now gives us:

Proposition 6.2. The moduli space $\mathcal{M}$ is a smooth hyperkähler manifold; the tangent space at $[A]$ is isomorphic to the $L^{2}$-kernel of $d_{A}^{+} \oplus d_{A}^{*}$ acting on $\Omega^{1}(\mathfrak{s u}(E))$. It has dimension $8 k-4$.

Proof of proposition 6.1. First, we have to understand the behaviour of the Laplacian $\Delta_{A}$ acting on sections of $\operatorname{End}(E)$. We want to prove that it is Fredholm. This property is not changed by a perturbation in $\Omega_{\delta}^{1}$ (this adds to $\Delta_{A}$ a compact operator), and we can therefore restrict to the case when $A=A_{0}$ on $r \geq R$. On this domain $r \geq R$, the Laplacian preserves the decomposition $u_{\Gamma} \oplus u_{\perp}$.

The case of $u_{\perp}$ is easier: since we have seen that $\left\|\nabla_{A_{0}}^{2} u\right\|_{L_{2-2 / p+\delta}^{p}}$ controls $\|u\|_{L_{2-2 / p+\delta}^{p}}$, it follows that $A_{0}-\Gamma$, which is $O\left(r^{-1}\right)$, is small if $R$ is big enough; therefore lemma 2.3 proves that $\nabla_{A}$ is an isomorphism on $r \geq R$ for the Neumann boundary condition (the same is true for Dirichlet boundary condition).

The case of $u_{\Gamma}$ is more complicated, but can be reduced to standard theory: recall that $u_{\Gamma}$ is torus invariant, so that the operator now reduces to an operator on $\mathbb{R}^{2}$; the action of $\Delta_{\Gamma}$ on off-diagonal coefficients (which exist only when $\Gamma$ is trivial) is by

$$
\frac{1}{r^{2}}\left(-\left(r \partial_{r}\right)^{2}+\left(\partial_{\theta}^{2} \pm 2 i \alpha\right)^{2}+|\mu|^{2}\right)
$$

and the action on diagonal coefficients is the standard Laplacian on $\mathbb{R}^{2}$ (that we obtain by making $\alpha=\mu=0$ in the previous formula); now $r^{2} \Delta_{A}$ becomes the translation invariant Laplacian

$$
-\partial_{t}^{2}-\left(\partial_{\theta}^{2} \pm 2 i \alpha\right)^{2}+|\mu|^{2}
$$

on the conformal cylinder $\mathbb{R}_{+} \times S^{1}$, so that standard theory [16] now applies: such operator (say, with Dirichlet boundary condition on $r=R$ ) is Fredholm for all weights, except a discrete set of critical weights $\delta$ (they are characterized by the existence at infinity of solutions of type $\left.\exp (-\delta t) t^{k}\right)$; moreover, as the operator is self-adjoint, its index is 0 at the weight 0 if it is noncritical, or -1 for small positive weights if 0 is critical; in our situation, $u \in T_{1} \mathcal{G}$ corresponds to the decay $u \in L_{\delta-2 / p}^{p}$, and this becomes exactly the weight $\delta$ on the cylinder; there are two cases: if $\alpha$ or $\mu$ is non zero (off-diagonal coefficients), then the weight 0 is not critical, and the operator remains Fredholm for nearby $\delta$, with index 0 : actually is is an isomorphism, because it easy to verify that is has no kernel; if $\alpha$ and $\mu$ are 
zero, then the Laplacian has index -1 for small weights $\delta>0$, so that it becomes an isomorphism if we add the possibility to consider solutions $u$ of $\Delta u=v$ with $u$ having some nonzero limit at infinity (and this is exactly our definition of $\mathcal{G}$ ). All these results can also be checked by direct calculation, after decomposing $u$ into Fourier series along each circle.

Finally, we deduce from these considerations that the Laplacian $\Delta_{A_{0}}$ is an isomorphism $T_{1} \mathcal{G} \rightarrow L_{2-2 / p+\delta}^{p}$ for the Dirichlet boundary conditions on $r \geq R$, and gluing this isomorphism with a parametrix on the compact part, it follows that $\Delta_{A}$ is Fredholm on $T \times \mathbb{R}^{2}$.

In order to calculate the index, if $\Gamma$ is nontrivial, we have seen that the index is not changed if we modify $A$ so that $A=\Gamma$ near infinity; the index of a self-adjoint operator on a compact manifold is zero; by an excision principle, this has the consequence that the index comes only from the contribution at infinity; therefore, it is equal to the index of the operator $\Delta_{\Gamma}$ acting on the trivial bundle $\mathfrak{s u}\left(\mathbb{C}^{2}\right)$; now this operator is completely explicit: on the $u_{\perp}$ component, it is an isomorphism, and on the $u_{\Gamma}$ component (that is, diagonal, torus invariant, matrices), it is simply the standard Laplacian in $\mathbb{R}^{2}$, and its index between the spaces that we have defined is again 0 , with 1-dimensional kernel and cokernel equal to constant diagonal matrices.

If $\Gamma$ is trivial, we cannot reduce to the operator of flat space, but we can reduce to $\Delta_{A_{0}^{\prime}}$, with $A_{0}^{\prime}$ the diagonal connection

$$
A_{0}^{\prime}=\chi(r) A_{0}+(1-\chi(r)) d,
$$

where $\chi(r)$ is a cutoff function which equals 1 for $r>R$ and 0 for $r<R-1$; then, as above, it is not difficult to prove that $\Delta_{A_{0}}^{\prime}$ is an isomorphism on non-diagonal components (and the operator on the diagonal components is the same as above).

Finally, the operator $\Delta_{A}$ has no kernel in $T_{1} \mathcal{G}$, since an element in the kernel would decompose $A$, which is impossible. This finishes the proof of the first part of the proposition.

If $A \in \mathcal{A}$ is an instanton, observe that the operator $d_{A}^{+} d_{A}^{*}$ acting on self-dual 2-forms, by the Weitzenböck formula, equals the Laplacian $\nabla_{A}^{*} \nabla_{A}$; this means that the above results remain true for $d_{A}^{+} d_{A}^{*}$, and we deduce that the operator

$$
d_{A}^{+} \oplus d_{A}^{*}: \Omega_{\delta}^{1} \longrightarrow L_{2-2 / p+\delta}^{p}
$$

is surjective; its kernel equals the kernel of the Laplacian $2\left(d_{A}^{+}\right)^{*} d_{A}^{+}+d_{A} d_{A}^{*}$; again one can prove (in particular using lemma 2.10) that this operator is Fredholm (for the weight $\delta$ ); remark that the $L^{2}$ condition corresponds to a critical weight (on diagonal components, where the operator is asymptotically the standard Laplacian of $\mathbb{R}^{2}$ ), when $\Omega_{\delta}^{1}$ corresponds to a slightly greater weight; nevertheless, it remains true that the $L^{2}$-kernel equals the kernel for slightly greater weights (the possible new solutions in the kernel at the critical weight are never $L^{2}$ ).

Proof of proposition 6.2. It remains only to calculate the dimension, which, by proposition 6.1, is the index of the operator $d_{A}^{+} \oplus d_{A}^{*}$. If the limit flat connection $\Gamma$ 
is non trivial, this is simple to calculate by comparison to the same operator for $\Gamma$ : actually, by the excision principle,

$$
\operatorname{ind}\left(d_{A}^{+} \oplus d_{A}^{*}\right)=\operatorname{ind}\left(d_{\Gamma}^{+} \oplus d_{\Gamma}^{*}\right)+8 k ;
$$

now for the flat connection $\Gamma$, the operator $d_{\Gamma}^{+} \oplus d_{\Gamma}^{*}$ has no kernel (by the Weitzenböck formula), but its cokernel equals the cokernel of the operator $d_{\Gamma}^{*} d_{\Gamma}+d_{\Gamma}^{+} d_{\Gamma}^{*}$ acting on $\Omega^{0}(\mathfrak{s u}(E)) \oplus \Omega_{+}^{2}(\mathfrak{s u}(E))=\mathbb{R}^{4} \otimes \mathfrak{s u}(E)$; we have seen above that the cokernel of this operator on $\mathfrak{s u}(E)$ is the $L^{2}$-orthogonal of constant, diagonal matrices. This proves the formula for the index.

If $\Gamma$ is trivial, the same result holds, but one must compare with the operator $d_{A_{0}^{\prime}}^{+} \oplus d_{A_{0}^{\prime}}^{*}$ defined in (47).

Fibration structure. It was shown in [14] that the moduli space of rank two holomorphic vector bundles over $T \times \mathbb{P}^{1}$ with trivial determinant and instanton number $k$ contains an open set $\mathcal{M}_{k}^{*}$ (corresponding to the so-called regular bundles) which has the structure of a fibration:

$$
\mathbb{T} \cdots \mathcal{M}_{k}^{*} \rightarrow \Sigma_{k}
$$

The fibres are complex tori of complex dimension $2 k-1$, and the base can be interpreted as the set of rational maps $\mathbb{P}^{1} \rightarrow \mathbb{P}^{1}$ of degree $k$, so that $\operatorname{dim} \Sigma_{k}=2 k+1$.

Fixing the splitting of $\mathcal{E}$ at $T_{\infty}$, i.e. fixing the asymptotic state of the corresponding instanton connection $A$, amounts to fixing the value of these rational maps at $\infty \in \mathbb{P}^{1}$. Moreover, as we will see in the next section, fixing the residue of $A$ amounts to fixing the first derivative at $\infty \in \mathbb{P}^{1}$.

Therefore, according to theorem 0.2 , we conclude that $\mathcal{M}_{\left(k, \pm \xi_{0}, \mu\right)}$, the moduli space of $S U_{2}$ doubly-periodic instantons with fixed instanton number $k$, asymptotic states $\pm \xi_{0}$ and residue $\mu$ with the complex structure induced from the complex structure $I_{1}$ on $T \times \mathbb{R}^{2}$, is a fibration over $\Sigma_{\left(k, \pm \xi_{0}, \mu\right)}$, the space of rational maps $f: \mathbb{P}^{1} \rightarrow \mathbb{P}^{1}$ with fixed $f(w=\infty)$ and $f^{\prime}(w=\infty)$, with fibres given complex tori of dimension $2 k-1$.

Moreover, it is possible to show that the such fibres are lagrangian with respect to complex symplectic structure on $\mathcal{M}_{\left(k, \pm \xi_{0}, \mu\right)}$ induced from the complex symplectic structure $\omega_{I_{2}}+I_{1} \omega_{I_{3}}$ on $T \times \mathbb{C}$ (see [15] for the proof of a similar result for elliptic K3 and abelian surfaces).

An example: $\mathbf{k}=\mathbf{1}$. We shall now give an explicit model for the moduli space of doubly-periodic instantons with $k=1$; clearly, we also assume that $\xi_{0} \neq-\xi_{0}$ and $\mu \neq 0$.

Our approach is based on the observations made above, that is, we shall study the set of rational maps $f: \mathbb{P}^{1} \rightarrow \mathbb{P}^{1}$ of degree 1 ; in a neighbourhood of $\infty \in \mathbb{P}^{1}$, such maps can be written as follows:

$$
f(w)=\frac{w+b}{c w+d}, \quad \text { where } w=0 \text { corresponds to } \infty \in \mathbb{P}^{1} .
$$


As we discussed above, we must still fix $f(0)$ and $f^{\prime}(0)$. This means that $b / d$ and $(d-c b) / d^{2} \neq 0$ are fixed. Thus, $\Sigma_{\left(1, \pm \xi_{0}, \mu\right)}=\mathbb{C}$, so that $\mathcal{M}_{\left(k, \pm \xi_{0}, \mu\right)}$ is an elliptic fibration over $\mathbb{C}$.

Actually, one can say more: there is an action of $T \times \mathbb{C}$ on the moduli space (by translations), so the moduli space is exactly $T \times \mathbb{C}$, and the metric is flat.

\section{Nahm transform}

We now shift our attention to the Nahm transform of doubly-periodic instanton connections [13]. Note that this transform was defined in [13] only for instantons such that the restriction of the underlying holomorphic bundle to a generic torus is $L_{\xi} \oplus L_{-\xi}$ (this is what we called the semisimple case). In this part, we shall restrict to this case.

Throughout this part, we assume familiarity with [13], but let us quickly recall how Nahm transform is defined. Given an instanton $A$ on a $S U_{2}$-bundle $E$ on $T \times \mathbb{R}^{2}$, one may twist $A$ by a flat connection on $T$; these twists $A_{\xi}$ are parameterized by $\xi \in \hat{T}$. Now there is a coupled Dirac operator

$$
D_{A_{\xi}}: \Gamma\left(S^{+} \otimes E\right) \longrightarrow \Gamma\left(S^{-} \otimes E\right)
$$

and one can show that the bundle of $L^{2}$-cokernels of $D_{A_{\xi}}$ is a rank $k$ vector bundle $V$ over $\hat{T} \backslash\left\{ \pm \xi_{0}\right\}$; there is a natural connection $B$ on $V$ obtained by projection, and one can define an endomorphism $\Phi$ of $V$ by taking an element $\beta \in \operatorname{ker} D_{A_{\xi}}^{*}$ to the projection of $w \beta$ on this kernel; the pair $(B, \Phi)$ satisfies Hitchin's equations on $\hat{T} \backslash\left\{ \pm \xi_{0}\right\}$.

From the holomorphic point of view, the picture is very clear: the spinor bundle $S$ is identified $\Lambda^{0, *}$, so that the $L^{2}$-kernel of $D_{A_{\xi}^{*}}^{*}$ is exactly the $L^{2}$-kernel of $\bar{\partial}_{A_{\xi}} \oplus \bar{\partial}_{A_{\xi}}^{*}$ on $\Omega^{0,1} \otimes E$. It can be proven that this $L^{2}$-kernel coincides with $H^{1}\left(T \times \mathbb{P}^{1}, \mathcal{E} \otimes L_{\xi}\right)$, where $\mathcal{E}$ is the holomorphic extension of $A$ on $T \times \mathbb{P}^{1}$; this provides a holomorphic extension of $V$ on the whole $\hat{T}$; this extension has degree -2 , as can be checked by Riemann-Roch theorem for families. Moreover, there is a natural interpretation for the Higgs field: one has the identification

$$
V_{\xi}=H^{1}\left(T \times \mathbb{P}^{1}, \mathcal{E} \otimes L_{\xi}\right)=\bigoplus_{w \in \mathbb{C}} H^{0}\left(T_{w}, E \otimes L_{\xi}\right)
$$

where of course there is only a finite number of points $w \in \mathbb{C}$ (actually $k$, counted with multiplicity) such that $H^{0}\left(T_{w}, E \otimes L_{\xi}\right) \neq 0$. Now the Higgs field $\Phi$ is multiplication by $w$ on $H^{0}\left(T_{w}, E \otimes L_{\xi}\right)$. From this description, one can see that the Higgs field has a simple pole at $\pm \xi_{0}$ with semisimple residue, and the residue has only one nonzero eigenvalue if $\xi_{0} \neq-\xi_{0}$, two otherwise.

We first study how the new asymptotic parameters of doubly-periodic instantons introduced in Part II behave under Nahm Transform. This will prepare the way for the proof of theorem 0.5 , our last result. 


\section{Asymptotic parameters}

Following the general philosophy that the Nahm Transform is a sort of nonlinear Fourier Transform, it is reasonable to expect the asymptotic behaviour of the instanton to be translated into further singularity data for the Higgs field.

Recall that $\left.\mathcal{E}\right|_{T_{\infty}}=L_{\xi_{0}} \oplus L_{-\xi_{0}}$. From (48) we deduce a holomorphic splitting of $V$ on a small neighbourhood of $\pm \xi_{0}$ :

$$
V_{\xi}=B_{\xi} \oplus R_{\xi}
$$

where $B_{\xi}$ corresponds to the points in $\mathbb{C}$ that remain bounded as $\xi \rightarrow \xi_{0}$ and $R \xi$ corresponds to the points that go off to infinity. Clearly, $B \xi$ approaches the kernel of $\operatorname{Res}_{ \pm \xi_{0}} \Phi$ as $\xi \rightarrow \xi_{0}$, while $R_{\xi}$ approaches the eigenspace of the nontrivial eigenvalues of the residue. This is the decomposition used in theorem 0.4 , which we will now prove.

We first prove the statement concerning the residues. The argument to establish the statement concerning the limiting holonomy is much more technical, and will involve a series of lemmas.

Residues. Let $\rho=r^{-1}$ and let $w^{\prime}=w^{-1}=\rho e^{-i \theta}$ be a coordinate near $\infty \in \mathbb{P}^{1}$. Clearly, the holomorphic structure on the restriction $\left.E\right|_{T_{w^{\prime}}}$ is given by the $(0,1)$-part of the $\left.A\right|_{T_{w^{\prime}}}$. Rewriting equation (34) in terms of $w^{\prime}$, we obtain:

$$
\left.\bar{\partial}_{A}\right|_{T_{w^{\prime}}}=\bar{\partial}+\left(\begin{array}{cc}
\lambda & 0 \\
0 & -\lambda
\end{array}\right) d \bar{z}+\left(\begin{array}{cc}
\mu & 0 \\
0 & -\mu
\end{array}\right) w^{\prime} d \bar{z}+O\left(\rho^{2}\right)
$$

so that:

$$
\left.\frac{d}{d w^{\prime}}\left(\left.\bar{\partial}_{A}\right|_{T_{w^{\prime}}}\right)\right|_{w^{\prime}=0}=\left(\begin{array}{cc}
\mu & 0 \\
0 & -\mu
\end{array}\right) .
$$

In other words, the residue $\mu$ can be regarded as the infinitesimal variation of the holomorphic bundle $\left.\mathcal{E}\right|_{T_{w}}$ at $w=\infty$.

Since for every $w^{\prime}$ sufficiently close to $\infty \in \mathbb{P}^{1}$ we can assume that $\left.E\right|_{T_{w^{\prime}}}=L_{\xi\left(w^{\prime}\right)} \oplus L_{-\xi\left(w^{\prime}\right)}$, the above expression implies that:

$$
\left.\frac{d}{d w^{\prime}} \xi\left(w^{\prime}\right)\right|_{w^{\prime}=0}=\mu
$$

The eigenvalue of $\Phi$ going to infinity is $w(\xi)=1 / w^{\prime}(\xi)$ by (48); the first statement of theorem 0.4 follows.

Limiting holonomy. Let us now look at the coupled Dirac Laplacian $\Delta_{A_{\xi}}$ acting on sections of $S^{+} \otimes E$; since $A$ is an instanton, we have that $D_{A_{\xi}}^{*} D_{A_{\xi}}=\nabla_{A_{\xi}}^{*} \nabla_{A_{\xi}}$, i. e. the Dirac Laplacian coincides with the trace Laplacian. This Laplacian is invertible in $L^{2}$ for $\xi \neq \pm \xi_{0}$ (see [13]; this is also a consequence of the lemmas below), and we note its inverse by $G_{A_{\xi}}$. Such inverse is useful to produce harmonic representative of elements of $H^{1}\left(T \times \mathbb{P}^{1}, \mathcal{E} \otimes L_{\xi}\right)$. Indeed, if we have a compactly 
supported $(0,1)$-form $\beta$ with values in $E$ such that $\bar{\partial}_{A_{\xi}} \beta=0$, then the $L^{2}$-harmonic representative of the class $[\beta]$ is given by

$$
\beta-\bar{\partial}_{A_{\xi}} G_{A_{\xi}} \bar{\partial}_{A_{\xi}}^{*} \beta .
$$

We now want to understand the inverse $G_{A_{\xi}}$ when $\xi$ approaches the asymptotic states $\pm \xi_{0}$. For simplicity, assume that $\xi_{0}=0$ in the next three lemmas; the general case can be obtained by substituting $\xi-\xi_{0}$ for $\xi$ in the expressions below.

We know from theorem 0.1 , where $\lambda=\xi$ :

$$
A_{\xi}=\left(A_{0}\right)_{\xi}+a \text { with }|a|=O\left(r^{-1-\epsilon}\right)
$$

and

$$
\begin{gathered}
\left(A_{0}\right)_{\xi}=d+i\left(\begin{array}{cc}
\alpha & 0 \\
0 & -\alpha
\end{array}\right) d \theta+i\left(\begin{array}{cc}
\lambda_{1} d x+\lambda_{2} d y & 0 \\
0 & -\lambda_{1} d x-\lambda_{2} d y
\end{array}\right)+ \\
\quad \frac{i}{r}\left(\begin{array}{cc}
\mu_{1} d x+\mu_{2} d y & 0 \\
0 & -\mu_{1} d x-\mu_{2} d y
\end{array}\right) .
\end{gathered}
$$

We assume also that either $\mu_{1}$ or $\mu_{2}$ is nonzero; however, the proofs below will also work if $\mu_{1}=\mu_{2}=0$, but $\alpha \neq 0$.

Lemma 7.1. Let $\sigma$ is a section of $E \rightarrow T \times \mathbb{C}$; if $\lambda$ is sufficiently small and $|w|$ is large enough, then:

$$
\int_{T_{w}}\left|\nabla_{\left(A_{0}\right)_{\xi}} \sigma\right|^{2} \geq\left|\lambda+\frac{\mu}{w}\right|^{2} \int_{T_{w}}|\sigma|^{2} .
$$

Proof. Consider the Fourier expansion $\sigma=\Sigma \sigma_{n m} e^{i(n x+m y)}$. Then on the torus $T_{w}$, we have:

$$
\begin{aligned}
\int_{T_{w}}|\nabla \sigma|^{2} & =\int_{T_{w}}\left|\left(\partial_{x}+i \lambda_{1}+i \frac{\mu_{1}}{|w|}\right) \sigma\right|^{2}+\left|\left(\partial_{y}+i \lambda_{2}+i \frac{\mu_{2}}{|w|}\right) \sigma\right|^{2} \\
& =\sum\left|n+i m+\lambda+\frac{\mu}{|w|}\right|^{2}\left|\sigma_{n m}\right|^{2} .
\end{aligned}
$$

However, under the hypothesis above,

$$
\left|n+i m+\lambda+\frac{\mu}{|w|}\right| \geq\left|\lambda+\frac{\mu}{w}\right|
$$

for all $n, m$, which proves the lemma.

Lemma 7.2. Let $\sigma$ is a section of $E \rightarrow T \times \mathbb{C}$; if $\lambda$ is sufficiently small and $R$ is large enough, then:

$$
\int_{r \geq R}\left|\nabla_{\left(A_{0}\right)_{\xi}} \sigma\right|^{2} \geq c|\mu|^{2} \int_{r \geq R} \frac{|\sigma|^{2}}{r^{2}} .
$$


Proof. First remark that if

$$
\left|\frac{\lambda w}{\mu}\right| \geq 2 \quad \text { or } \quad\left|\frac{\lambda w}{\mu}\right| \leq \frac{1}{2}
$$

then we get

$$
\left|\lambda+\frac{\mu}{w}\right|=\frac{|\mu|}{|w|}\left|1+\frac{\lambda w}{\mu}\right| \geq \frac{1}{2} \frac{|\mu|}{|w|} ;
$$

using the previous lemma, we deduce that the wanted estimate holds over any torus $T_{w}$ such that $|w|=r$ satisfies (51), that is, the estimate of the lemma holds if we restrict outside the region

$$
\frac{1}{2} \frac{|\mu|}{|\lambda|} \leq r \leq 2 \frac{|\mu|}{|\lambda|}
$$

Actually, we claim that if the estimate of the lemma is satisfied outside this region, then it must be satisfied everywhere. Indeed, one has the inequality for any function $f: \mathbb{R}^{2} \rightarrow \mathbb{R}$, and a constant $c$ independent of $\rho$,

$$
\int_{\rho \leq r \leq 2 \rho} \frac{f^{2}}{r^{2}} \leq c \cdot\left(\int_{2 \rho \leq r \leq 4 \rho} \frac{f^{2}}{r^{2}}+\int_{\rho \leq r \leq 4 \rho}\left|\partial_{r} f\right|^{2}\right)
$$

and the lemma follows by applying (52) to $f=|\sigma|$ and $\rho=|\lambda / \mu|$. The proof of (52) is left to the reader.

Note that an estimate similar to (50) remains valid if $\mu=0$, but $\alpha \neq 0$. In fact, the proof is even simpler, since one has the estimate:

$$
\int_{r=R}\left|\nabla_{\left(A_{0}\right)_{\xi}} \sigma\right|^{2} \geq \frac{|\alpha|^{2}}{r^{2}} \int_{r \geq R}|\sigma|^{2}
$$

from which one immediately obtains:

$$
\int_{r \geq R}\left|\nabla_{\left(A_{0}\right)_{\xi}} \sigma\right|^{2} \geq|\alpha|^{2} \int_{r \geq R} \frac{|\sigma|^{2}}{r^{2}} .
$$

Lemma 7.3. The solution of the Poisson equation $\Delta_{A_{\xi}} u=v$ satisfies:

$$
\begin{gathered}
\left\|r^{-1} u\right\|_{L^{2}}+\left\|\nabla_{A_{\xi}} u\right\|_{L^{2}} \leq c\|r v\|_{L^{2}} \\
\text { and }|\xi|^{2}\|u\|_{L^{2}}+|\xi| \cdot\left\|\nabla_{A_{\xi}} u\right\|_{L^{2}} \leq c\|r v\|_{L^{2}} \text {. }
\end{gathered}
$$

Proof. First, note that:

$$
\int\left|\nabla_{A_{\xi}} \sigma\right|^{2} \geq c\left(|\xi|^{2} \int|\sigma|^{2}+\int \frac{|\sigma|^{2}}{r^{2}}\right) .
$$

Near infinity, this a consequence of lemma 7.2 and of the fact that $A=A_{0}+$ $O\left(r^{-1-\epsilon}\right)$. Globally, the estimate follows from the Poincaré-type inequality:

$$
\int_{r \leq R}|\sigma|^{2} \leq c\left(\int_{r \leq R}|\nabla \sigma|^{2}+\int_{R / 2 \leq r \leq R}|\sigma|^{2}\right) .
$$


To prove the lemma itself, we have that:

$$
\begin{aligned}
\left\|\nabla_{A_{\xi}} u\right\|_{L^{2}}^{2} & =\int\left\langle\Delta_{A_{\xi}} u, u\right\rangle=\int\langle v, u\rangle \leq \\
& \leq\|r v\|_{L^{2}}\left\|r^{-1} u\right\|_{L^{2}} \leq c\|r v\|_{L^{2}}\left\|\nabla_{A_{\xi}} u\right\|_{L^{2}}
\end{aligned}
$$

by (54). Thus, we conclude that $\left\|\nabla_{A_{\xi}} u\right\|_{L^{2}} \leq c\|r v\|_{L^{2}}$, and again by (54) we have $\left\|r^{-1} u\right\|_{L^{2}} \leq c\|r v\|_{L^{2}}$. The second estimate is obtained in a similar way.

Proof of theorem 0.4. Let us first analyze the behaviour of the harmonic metric on the local sub-bundle $B \hookrightarrow V$ with fibers given by $B_{\xi}$. Let $\beta$ be a section of $B$. Then, for each $\xi \neq \xi_{0}$, we know from (48) that $\beta(\xi)$ can be represented as a section of $\Lambda^{0,1} E \otimes L_{\xi}$ supported on $r \leq R$ for some $R$ sufficiently large. Furthermore, its harmonic representative in $H^{1}\left(T \times \mathbb{C}, E \otimes L_{\xi}\right)$ is given by $\beta(\xi)-\bar{\partial}_{A_{\xi}} G_{A_{\xi}} \bar{\partial}_{A_{\xi}}^{*} \beta(\xi)$. By lemma 7.3, we have:

$$
\left\|\bar{\partial}_{A_{\xi}} G_{A_{\xi}} \bar{\partial}_{A_{\xi}}^{*} \beta(\xi)\right\|_{L^{2}} \leq c\left\|r \bar{\partial}_{A}^{*} \beta(\xi)\right\|_{L^{2}} \leq c R\left\|\bar{\partial}_{A}^{*} \beta(\xi)\right\|_{L^{2}}
$$

which remains bounded even as $\xi \rightarrow \xi_{0}$. This means that the limit

$$
\beta\left(\xi_{0}\right)=\lim _{\xi \rightarrow \xi_{0}} \beta(\xi)
$$

has a square-integrable harmonic representative, so that the harmonic metric restricted to the sub-bundle $B$ extends across $\pm \xi_{0}$.

Now let $R \hookrightarrow V$ be a local sub-bundle with fibers given by $R_{\xi}$; remind that near infinity, we have $\left.\mathcal{E}\right|_{T_{w}}=L_{\xi(w)} \oplus L_{-\xi(w)}$; take a section $\beta(\xi)$ of $R_{\xi}$ coming by (48) from sections of $\left.\mathcal{E}\right|_{T_{w(\xi)}} \otimes L_{\xi}$ converging to a section of $\left.\mathcal{E}\right|_{T_{\infty}} \otimes L_{\xi_{0}}=L_{2 \xi_{0}} \oplus \mathbb{C}$. Here we have to be more specific: say that a section $\sigma \in H^{0}\left(T_{w(\xi)}, \mathcal{E} \otimes L_{\xi}\right)$ corresponds to the class in $H^{1}\left(T \times \mathbb{P}^{1}, \mathcal{E} \otimes L_{\xi}\right)$ represented by the $(0,1)$-current

$$
\sigma(z) \delta_{w(\xi)}(w) d \bar{w}
$$

where $\delta_{w(\xi)}$ is the Dirac function at the point $w(\xi)$. From this description, we see that, for each $\xi \neq \xi_{0}$, the representative $\beta(\xi)$ can be chosen with compact support near $r=|w(\xi)|$, and bounded in $L^{1,2}$. Now lemma 7.3 gives, as above,

$$
\left\|\bar{\partial}_{A_{\xi}} G_{A_{\xi}} \bar{\partial}_{A_{\xi}}^{*} \beta(\xi)\right\|_{L^{2}} \leq c\left\|r \bar{\partial}_{A}^{*} \beta(\xi)\right\|_{L^{2}} \leq \frac{c}{\left|\xi-\xi_{0}\right|}\left\|\bar{\partial}_{A}^{*} \beta(\xi)\right\|_{L^{2}}
$$

This means that the norm of the harmonic representative of $\beta(\xi)$ is bounded by $\left|\xi-\xi_{0}\right|^{-1}$.

This result must be interpreted, since (55) actually does not extend to $w=\infty$, so that our $[\beta(\xi)]$ is not a section of $R$ which extends over the puncture $\xi_{0}$. There are two changes to make; first, note that a $(0,1)$-form smooth on $\mathbb{P}^{1}$ near infinity is $d \bar{w} / \bar{w}^{2}$, so we see that we must consider $\beta(\xi) / \bar{w}(\xi)^{2}$ instead of $\beta(\xi)$. The second change to be made is that we want $\beta(\xi)$ holomorphic in $\xi$. This involves a constraint on the choice of $\sigma$ : from the growth of the holomorphic sections of $\mathcal{E}$ at infinity studied in Sect. 5, it follows that $|\sigma| \sim|w(\xi)|^{\alpha}$, and we can finally conclude that the norm of a holomorphic section of $R$ is bounded by $\left|\xi-\xi_{0}\right|^{1-\alpha}$. 
From these results, it follows that the harmonic metric of the Higgs bundle $V$ extends on $B$, and is bounded by $\left|\xi \pm \xi_{0}\right|^{1 \pm \alpha}$ on $R$. This gives a bound $1 \pm \alpha$ for the weights of the parabolic structure of $V$. However, the "parabolic degree" of the bundle must be zero, and $V$ has degree -2 , so that the weights must be exactly equal to $1 \pm \alpha$.

Reformulating the Nahm transform theorem. Together with [12, 13], theorem 0.4 allows us to state a complete version of the Nahm transform theorem, including the new asymptotic parameters defined in Part II:

Theorem 7.4. The Nahm transform is a correspondence between the following objects:

- $S U(2)$ doubly-periodic instantons with instanton number $k>0$ and asymptotic parameters $\left( \pm \xi_{0}, \alpha, \mu\right)$;

- rank $k$ logarithmic Higgs bundles with harmonic metric over $\hat{T}$ with singularity behaviour as described in theorem 0.4.

Remark 7.5. At the algebraic level, the behaviour of the harmonic metric in theorem 0.4 means, by Simpson's correspondence [22], that the holomorphic bundle $(V, \Phi)$ is a stable parabolic Higgs bundle on $\hat{T}$; here there is a slight problem because all the weights should belong to an interval of length 1 , so we need to be more precise.

Remind the decomposition (49) of the bundle $V$ near $\pm \xi_{0}$, and define a modification $W$ of $V$ near the $\pm \xi_{0}$ by

$$
W=B \oplus R \otimes \mathcal{O}\left( \pm \xi_{0}\right),
$$

and $W$ is isomorphic to $V$ outside the punctures; the degree of $W$ is now zero, and the harmonic metric behaves like $\left|\xi \pm \xi_{0}\right|^{ \pm \alpha}$ on $R\left( \pm \xi_{0}\right)$. This means that $W$ has a parabolic structure at $\pm \xi_{0}$, with weights $(0, \pm \alpha)$ at $\pm \xi_{0}$ and $\pm \alpha$ of multiplicity 1 , and the existence of the harmonic metric is now equivalent to the parabolic stability of $W$.

\section{The hyperkähler property}

Our final task is to prove that the Nahm transform of doubly-periodic instantons define a hyperkähler isometry between $\mathcal{M}$, the moduli space of doubly-periodic instanton constructed in Sect. 6, and $\hat{\mathcal{M}}$, the moduli space of meromorphic Higgs pairs satisfying the conditions of theorem 7.4. To do that, we shall follow the following strategy. First, we compute the derivative of the map:

$$
\begin{aligned}
N: \mathcal{M} & \longrightarrow \hat{\mathcal{M}} \\
A & \mapsto(B, \Phi)
\end{aligned}
$$

defined by the Nahm transform, verifying that it is indeed well-defined. We then show that $D_{[A]} N$ preserves the three complex structures in each space. The last step is to show that $D_{[A]} N$ preserve the metrics in each space. 
Computing the derivative. Recall the definition of the tangent space $T_{[A]} \mathcal{M}$ at the gauge equivalence class of an instanton $A$ can be characterized as follows:

$$
T_{[A]} \mathcal{M}=\left\{a \in L^{2}\left(\Omega^{1}{ }_{\mathfrak{s u}}(E)\right) \text { s.t. } \begin{array}{l}
\text { (i) } d_{A}^{*} a=0 \\
\text { (ii) } d_{A}^{+} a=0
\end{array}\right\}
$$

The 1-form $a$ is regarded as a infinitesimal variation of the instanton connection $A$, inducing a 1-parameter family of connections $A_{t}=A+t a$, which are anti-self-dual up to first order.

Now let $\left\{\Psi(\xi)^{j}\right\}_{j=1}^{k}$ be an orthonormal base for coupled adjoint Dirac operator $\operatorname{ker} D_{A_{\xi}}^{*}$. In order to compute the derivative $D_{[A]} N$, we must understand the infinitesimal change on harmonic spinors induced by the infinitesimal change on the instanton. We are looking for negative spinors $\varphi(\xi)^{j}$ such that the 1-parameter family $\Psi_{t}(\xi)^{j}=\Psi(\xi)^{j}+t \cdot \varphi(\xi)^{j}$ satisfies $D_{\left(A_{\xi}\right)_{t}}^{*} \Psi_{t}(\xi)^{j}=0$ up to first order. In other words,

$$
\left.\frac{d}{d t} D_{\left(A_{\xi}\right)_{t}}^{*} \Psi_{t}(\xi)^{j}\right|_{t=0}=D_{A_{\xi}}^{*} \varphi(\xi)^{j}+a \bullet \Psi(\xi)^{j}=0
$$

where $\bullet$ means Clifford multiplication. Therefore, the infinitesimal variations on harmonic spinors are given by:

$$
\varphi(\xi)^{j}=-D_{A_{\xi}} G_{A_{\xi}}\left(a \bullet \Psi(\xi)^{j}\right) .
$$

Recall from [13] that the Nahm transformed Higgs pair is defined as follows:

$$
B(\xi)^{i j}=\left\langle\Psi(\xi)^{i}, \hat{d} \Psi(\xi)^{j}\right\rangle \text { and } \Phi(\xi)^{i j}=\left\langle\Psi(\xi)^{i}, w \Psi(\xi)^{j}\right\rangle d \xi
$$

where $\hat{d}$ means differentiation with respect to $\xi$, the coordinate on the dual torus $\hat{T}$, and the inner products are taken in $L^{2}\left(E \otimes S^{-}\right)$. Thus, the infinitesimal change in the Nahm transformed Higgs pair $(B, \Phi)$ is given by:

$$
\begin{aligned}
b(\xi)^{i j} & =\left.\frac{d}{d t}\left\langle\Psi_{t}(\xi)^{j}, \hat{d} \Psi_{t}(\xi)^{j}\right\rangle\right|_{t=0}= \\
& =\left\langle G_{A_{\xi}} \Psi(\xi)^{i}, \Omega \bullet a \bullet \Psi(\xi)^{j}\right\rangle-\left\langle\Omega \bullet a \bullet \Psi(\xi)^{i}, G_{A_{\xi}} \Psi(\xi)^{j}\right\rangle
\end{aligned}
$$

and

$$
\begin{aligned}
\phi(\xi)^{i j} & =\left.\frac{d}{d t}\left\langle\Psi_{t}(\xi)^{j}, w \Psi_{t}(\xi)^{j}\right\rangle\right|_{t=0}= \\
& =\left\langle G_{A_{\xi}} \Psi(\xi)^{i}, d w \bullet a \bullet \Psi(\xi)^{j}\right\rangle d \xi
\end{aligned}
$$

where $\Omega=i\left(d \xi_{1} d z_{1}+d \xi_{2} d z_{2}\right)$ is the curvature of the Poincaré bundle over $T \times \hat{T}$. 
The tangent space $T_{[(B, \Phi)]} \hat{\mathcal{M}}$ at the gauge equivalence class of a Higgs pair $(B, \Phi)$, can described as follows (see for instance [10]):

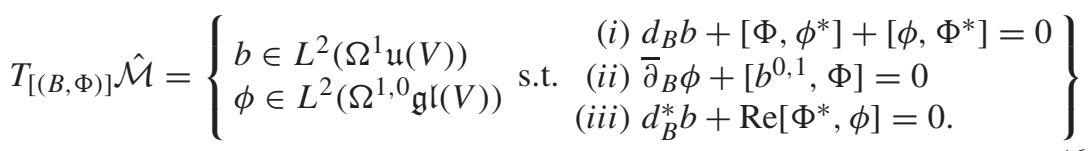

Again, $(b, \phi)$ define a 1-parameter family of pairs $\left(B_{t}=B+t b, \Phi_{t}=\Phi+t \phi\right)$ which satisfy Hitchin's equations up to first order.

Therefore, it is clear from (59) and (60) that the pair $(b, \phi)$ satisfies the linearized Hitchin's equations ( $($ i) and (ii) in (61)).

We must only verify that $(b, \phi)$ are transversal to infinitesimal changes in $(B, \Phi)$ arising from infinitesimal gauge transformations, i.e. must check equation (iii) in (61). To do that, denote by $\tilde{B}$ and $\tilde{b}$ the $\left(\mathbb{R}^{2}\right)^{*}$-invariant 1 -forms on $\hat{T} \times\left(\mathbb{R}^{2}\right)^{*}$ obtained from $(B, \Phi)$ and $(b, \phi)$, respectively. Clearly, $\tilde{B}$ is anti-self-dual and

$$
d_{B}^{*} b+\operatorname{Re}\left[\Phi^{*}, \phi\right]=0 \Leftrightarrow d_{\tilde{B}}^{*} \tilde{b}=0
$$

The following result completes our first step towards the proof of theorem 0.5

Lemma 8.1. If $d_{A}^{*} a=0$, then $d_{\tilde{B}}^{*} \tilde{b}=0$.

Proof. See proposition 3.1 in [4].

Remark 8.2. Using the ideas above, one can easily compute the derivative of the inverse Nahm transform, thus showing that $N: \mathcal{M} \rightarrow \hat{\mathcal{M}}$ is a diffeomorphism. From Nakajima [19], we know that the diffeomorphism type of the moduli of Higgs bundles does not depend on the choice of the singularity data (residues and parabolic structure). Therefore, we conclude the diffeomorphism type of the moduli space of doubly-periodic instantons does not depend neither on the limiting holonomy $\alpha$ nor on the residue $\mu$. It would be interesting to determine whether the diffeomorphism type of $\mathcal{M}$ depends on the asymptotic states $\pm \xi_{0}$ (or equivalently, whether the diffeomorphism type moduli of Higgs bundles depend on the position of the singularities).

Commuting with the complex structures. Consider coordinates $\left(\xi_{1}, \xi_{2}, \omega_{1}, \omega_{2}\right)$ on $\left(\mathbb{R}^{4}\right)^{*}$, which are dual to $\left(z_{1}, z_{2}, w_{1}, w_{2}\right)$. Each of the complex structures (46) in $\mathbb{R}^{4}$ naturally induces a similar complex structures $\hat{I}_{j}$ on $\left(\mathbb{R}^{4}\right)^{*}$. Thus, we have maps:

$$
\Lambda^{1} \mathbb{R}^{4} \stackrel{I_{j}}{\rightarrow} \Lambda^{1} \mathbb{R}^{4} \text { and } \Lambda^{1}\left(\mathbb{R}^{4}\right)^{*} \stackrel{\hat{I}_{j}}{\rightarrow} \Lambda^{1}\left(\mathbb{R}^{4}\right)^{*}
$$

The complex structures on $\hat{\mathcal{M}}$ can be then defined as follows. As above, let $\tilde{b} \Lambda^{1}\left(\mathbb{R}^{4}\right)^{*}$ be the $\left(\mathbb{Z}^{2} \times \mathbb{R}^{2}\right)^{*}$-invariant 1 -form obtained from $(b, \phi)$. Then $\hat{I}_{j}(\tilde{b})$ is also a $\left(\mathbb{Z}^{2} \times \mathbb{R}^{2}\right)^{*}$-invariant 1 -form on $\left(\mathbb{R}^{4}\right)^{*}$, which can then be interpreted as an element of (61). It is easy to see that these coincide with the complex structures 
originally defined by Hitchin in [10]. Therefore, we have to show that the following diagram:

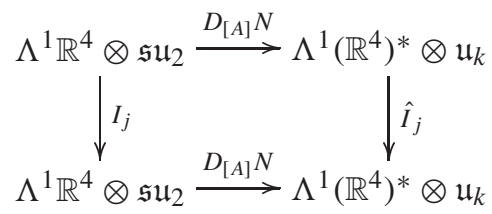

commutes. The horizontal maps are defined as follows:

$$
D_{[A]} N(a)=\tilde{b}=\left\langle G_{A_{\xi}} \Psi(\xi)^{i}, \widetilde{\Omega} \bullet a \bullet \Psi(\xi)^{j}\right\rangle-\left\langle\widetilde{\Omega} \bullet a \bullet \Psi(\xi)^{i}, G_{A_{\xi}} \Psi(\xi)^{j}\right\rangle
$$

with $\widetilde{\Omega}=i\left(d \xi_{1} d z_{1}+d \xi_{2} d z_{2}+d \omega_{1} d w_{1}+d \omega_{2} d w_{2}\right)$.

Each $I_{j}$ induces an isomorphism $l_{j}: \mathbb{R}^{4} \rightarrow \mathbb{C}^{2}$ satisfying the following commutative diagram:

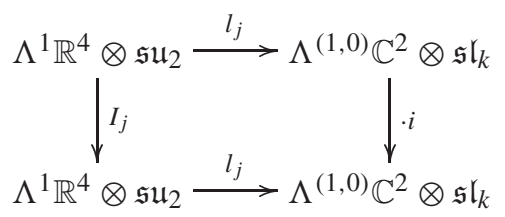

where the map on the left hand side is multiplication by $i=\sqrt{-1}$. Of course, a similar diagram holds for $\hat{l_{j}}:\left(\mathbb{R}^{4}\right)^{*} \rightarrow\left(\mathbb{C}^{2}\right)^{*}$.

The key point is to note that each map:

$$
\begin{gathered}
D_{[A]} N_{\mathbb{C}}=\hat{l_{j}} \circ D_{[A]} N \circ l_{j}^{-1}: \Lambda^{(1,0)} \mathbb{C}^{2} \rightarrow \Lambda^{(1,0)}\left(\mathbb{C}^{2}\right)^{*} \\
D_{[A]} N_{\mathbb{C}}(\alpha)=\left\langle G_{A_{\xi}} \Psi(\xi)^{i}, \widetilde{\Omega}_{\mathbb{C}} \bullet \bar{\alpha} \bullet \Psi(\xi)^{j}\right\rangle-\left\langle\widetilde{\Omega}_{\mathbb{C}} \bullet \alpha \bullet \Psi(\xi)^{i}, G_{A_{\xi}} \Psi(\xi)^{j}\right\rangle
\end{gathered}
$$

is $\mathbb{C}$-linear, where $\widetilde{\Omega}_{\mathbb{C}}=\hat{l}_{j} \times l_{j}(\widetilde{\Omega})$. Therefore, we conclude:

$$
\begin{aligned}
\hat{I}_{j}\left(D_{[A]} N(a)\right) & ={\hat{l_{j}}}^{-1} \circ(\cdot i) \circ \hat{l_{j}} \circ D_{[A]} N(a)=D_{[A]} N \circ l_{j}^{-1} \circ(\cdot i) \circ l_{j}(a)= \\
& =D_{[A]} N\left(I_{j}(a)\right)
\end{aligned}
$$

as desired.

The Nahm transform is an isometry. Again, this fact is actually a property of the underlying 4-dimensional transform. The calculations of Braam and van Baal [4] are quite precise and also apply to the present situation.

Recall that the metric on the instanton moduli space is given by the $L^{2}$ norm of the tangent vectors, that is:

$$
g\left(a_{1}, a_{2}\right)=\int_{T \times \mathbb{C}} \operatorname{Tr}\left(a_{1} \wedge * a_{2}\right)
$$

while the metric on the Higgs moduli space is given by

$$
\hat{g}\left(\left(b_{1}, \phi_{1}\right),\left(b_{2}, \phi_{2}\right)\right)=\int_{\hat{T}} \operatorname{Tr}\left(b_{1}^{*} b_{2}+\phi_{1} \phi_{2}^{*}\right)
$$


or, equivalently, in terms of the 4-dimensional 1-forms $\tilde{b_{1}}$ and $\tilde{b_{2}}$ :

$$
\hat{g}\left(\tilde{b_{1}}, \tilde{b_{2}}\right)=\int_{\mathbb{R}^{2}}^{*} \operatorname{Tr}\left(\tilde{b_{1}} \wedge * \tilde{b_{2}}\right)
$$

where integration is now done only with respect to the two coordinates on $\left(\mathbb{R}^{4}\right)^{*}$ on which $\tilde{b_{1}}$ and $\tilde{b_{1}}$ depend.

Let $(b, \phi)=D_{[A]} N(a)$; it is enough to show that:

$$
\hat{g}\left(D_{[A]} N(a),(b, \phi)\right)=g\left(a, D_{[A]} N^{-1}(b, \phi)\right)
$$

This can be done exactly as proposition 3.2 of [4].

Alternatively, we can reduce the isometry property to a purely algebraic statement as follows.

Fix the complex structure $I_{1}$ on $T^{2} \times \mathbb{R}^{2}$. The instanton moduli space $\mathcal{M}$ is then identified with the moduli space of $\alpha$-stable holomorphic vector bundles $\mathcal{E} \rightarrow T \times \mathbb{P}^{1}$ as a Kähler manifold. Moreover, its tangent space becomes identified with $H^{1}\left(T \times \mathbb{P}^{1}\right.$, End $\left.\mathcal{E}\right)$. One can define a complex symplectic structure on $\mathcal{M}$ via the bilinear pairing:

$$
H^{1}\left(T \times \mathbb{P}^{1}, \text { End } \mathcal{E}\right) \times H^{1}\left(T \times \mathbb{P}^{1}, \text { End } \mathcal{E}\right) \stackrel{\omega}{\rightarrow} H^{2}\left(T \times \mathbb{P}^{1}, \text { End } \mathcal{E}\right)=\mathbb{C}
$$

On the other hand, the moduli space of Higgs pairs $\hat{\mathcal{M}}$ becomes identified, as a Kähler manifold, with the moduli space of stable parabolic Higgs bundles. The tangent is then given by the hypercohomology $\mathbb{H}^{1}$ of the following complex of sheaves:

$$
\operatorname{ParEnd}(\mathcal{V}) \stackrel{[\cdot, \phi]}{\rightarrow} \Lambda^{1} \otimes \operatorname{ParEnd}(\mathcal{V})
$$

where $\operatorname{ParEnd}(\mathcal{V})$ is the sheaf of parabolic endomorphism of the holomorphic Higgs bundle $\mathcal{V}$, see [3] for a detailed explanation. A complex symplectic structure on $\hat{\mathcal{M}}$ can be defined via the bilinear pairing

$$
\mathbb{H}^{1} \times \mathbb{H}^{1} \stackrel{\hat{\omega}}{\rightarrow} \mathbb{H}^{2}=\mathbb{C}
$$

In order to show that the Nahm transform is an isometry, it is enough to prove that the holomorphic version of the Nahm transform (see [14]) preserves the bilinear pairings above. This is an algebraic statement, which one can hope to prove using spectral sequences. Indeed, as we mentioned before, the holomorphic version of the Nahm transform of doubly-periodic instantons is an example of a Fourier-Mukai transform, which usually preserves this type of pairings.

Acknowledgements. The second author would like to thank the École Polytechnique for its support, and Antony Maciocia for useful conversations. 


\section{References}

1. O. Biquard: Prolongement d'un fibré holomorphe hermitien à courbure $L^{p}$. Int. J. Math. 3, 441-453 (1992)

2. O. Biquard: Sur les fibrés paraboliques sur une surface complexe. J. London Math. Soc. 53 (2), 302-316 (1996)

3. O. Biquard: Fibrés de Higgs et connexions intégrables: le cas logarithmique (diviseur lisse). Ann. Scient. Éc. Norm. Sup. 30 (4), 41-96 (1997)

4. P. Braam, P. van Baal: Nahm's transformation for instanton. Commun. Math. Phys. 122, 267-280 (1989)

5. N. Buchdahl: Instantons on $n$ CP$^{2}$. J. Diff. Geom. 37, 669-687 (1993)

6. C. Ford, J. Pawlowski, T. Tok, A. Wipf: ADHM construction of instantons on the torus. Nucl. Phys. B 596, 387-414 (2001)

7. S. Donaldson: Instantons and geometric invariant theory. Commun. Math. Phys. 93, 453-460 (1984)

8. R. Fahlaoui, Y. Laszlo: Transformée de Fourier et stabilité sur les surfaces abéliennes. Compositio Math. 79, 271-278 (1991)

9. A. González-Arroyo, A. Montero: Self-dual vortex-like configurations in SU(2) YangMills theory. Phys. Lett. B442, 273-278 (1998)

10. N. Hitchin: The self-duality equations on a Riemann surface. Proc. London Math. Soc. 55, 59-126 (1987)

11. A. Kapustin, S. Sethi: The Higgs branch of impurity theories. Adv. Theor. Math. Phys. 3 (2), 571-591 (1998)

12. M. Jardim: Construction of doubly-periodic instantons. Preprint math.DG/9909069. Commun. Math. Phys. (to appear)

13. M. Jardim: Nahm transform of doubly-periodic instantons. Preprint math.DG/9910120

14. M. Jardim: Spectral curves and the Nahm transform of doubly-periodic instantons. Preprint math.AG/9909146

15. M. Jardim, A. Maciocia: A Fourier-Mukai approach to the spectral data for instantons. Preprint math.AG/0006054

16. R. Lockhart, R. McOwen: Elliptic differential operators on non-compact manifolds. Ann. Sc. Norm. Sup. Pisa 12 (4), 409-447 (1985)

17. A. Maciocia: Metrics on the moduli spaces of instantons over Euclidean 4-space. Commun. Math. Phys. 135, 467-482 (1991)

18. H. Nakajima: Monopoles and Nahm's equations. In: Lect. Notes Pure Appl. Math. 145, pp. 193-211. New York: Marcel Dekker 1993

19. H. Nakajima: Hyperkähler structures on the moduli spaces of parabolic Higgs bundles on Riemann surfaces. In: Lect. Notes Pure Appl. Math. 179, pp. 199-208. New York: Marcel Dekker 1996

20. J. Råde: Singular Yang-Mills fields. Local theory II. J. reine angew. Math. 456, 197-219 (1994)

21. C. Simpson: Constructing variations of Hodge structure using Yang-Mills theory and applications to uniformization. J. Amer. Math. Soc. 1, 867-918 (1988)

22. C. Simpson: Harmonic bundles on noncompact curves. J. Amer. Math. Soc. 3, 713-770 (1990) 